\title{
Partitioning graphs into paths or cycles of prescribed lengths
}

\author{
Colton Magnant and Kenta Ozeki
}

\begin{abstract}
In this paper, we consider the path (and cycle) partition problem for graphs with additional length restrictions. More specifically, we prove sufficient degree sum conditions for the vertices of a graph to be partitioned into paths, with fixed end vertices, such that these paths have approximately prescribed lengths. We also prove similar results for partitions into cycles of approximately prescribed lengths each containing a specified vertex.
\end{abstract}

AMS 2000 SUBJECT CLASSIFICATIONS: Primary 05C70; secondary 05C35. KEYWORDS AND PHRASES: Graph partition, degree sum, path length.

\section{Partition problem into paths or cycles}

Many researchers have considered the "path (or cycle) partition problem", that is, determining whether the vertices of a graph can be partitioned into paths or cycles (with some properties, mentioned after). In particular, we are interested in a $\sigma_{2}(G)$ condition which implies the existence of such a partition, where

$$
\sigma_{2}(G):=\min \{d(x)+d(y): x y \notin E(G)\}
$$

if $G$ is not a complete graph; otherwise let $\sigma_{2}(G):=+\infty$. For many related problems and results, we refer the reader to the path and cycle partition problem survey [6].

In this paper, we first concentrate on a partition into paths with some special properties. It is easily proved that if $\sigma_{2}(G) \geq n-t$ for a graph $G$ of order $n$, there exist $t$ disjoint paths $P_{1}, P_{2}, \ldots, P_{t}$ with $V(G)=\bigcup_{i=1}^{t} V\left(P_{i}\right)$. By considering the classical result on a hamilton cycle by Ore [13], we can obtain the following fact.

Theorem 1 (Ore [13]). Let $t$ be an integer with $t \geq 2$ and let $G$ be a graph of order $n$. If $\sigma_{2}(G) \geq n$, then for any $t$ vertices $x_{1}, x_{2}, \ldots, x_{t}$, there exists $t$ pairwise disjoint paths $P_{1}, P_{2}, \ldots, P_{t}$ such that $V(G)=\bigcup_{i=1}^{t} V\left(P_{i}\right)$ and $x_{i}$ is an end vertex of $P_{i}$. 
On the other hand, Johansson [9] gave a sharp $\delta(G)$ condition for the existence of a partition into paths with given order. Chen et al. extended Johansson's result, and we obtain the following $\sigma_{2}(G)$ condition as a corollary of it.

Theorem 2 (Chen et al. [2]). Let $t$ be an integer with $t \geq 2$ and also let $a_{1}, a_{2}, \ldots, a_{t}$ be integers with $a_{i} \geq 2$ for any $1 \leq i \leq t$. Let $G$ be a graph of order $n=\sum_{i=1}^{t} a_{i}$ and suppose $\sigma_{2}(G) \geq n-t+\lambda-2$, where $\lambda$ is the number of even integers in $\left\{a_{1}, a_{2}, \ldots, a_{t}\right\}$. Then there exist $t$ pairwise disjoint paths $P_{1}, P_{2}, \ldots, P_{t}$ such that $V(G)=\bigcup_{i=1}^{t} V\left(P_{i}\right)$ and $\left|P_{i}\right|=a_{i}$.

Enomoto and Ota considered the conditions from Theorems 1 and 2 at the same time, that is, a partition into paths with specified end vertices and given order. They also posed the following conjecture.

Conjecture 1 (Enomoto and Ota [7]). Let $t$ be an integer with $t \geq 2$ and let $a_{1}, a_{2}, \ldots, a_{t}$ be positive integers. Let $G$ be a graph of order $n=\sum_{i=1}^{t} a_{i}$. If $\sigma_{2}(G) \geq n+t-1$, for any $t$ vertices $x_{1}, x_{2}, \ldots, x_{t}$, then there exist $t$ vertex disjoint paths $P_{1}, P_{2}, \ldots, P_{t}$ such that $V(G)=\bigcup_{i=1}^{t} V\left(P_{i}\right), x_{i}$ is an end vertex of $P_{i}$ and $\left|P_{i}\right|=a_{i}$.

The sharpness of the aforementioned conjecture (if it is true) is given by $G_{1}=K_{t}+\left(K_{1} \cup K_{n-t-1}\right)$. Notice $\sigma_{2}\left(G_{1}\right)=t+(n-t-2+t)=n+t-2$. If the vertices of the $K_{t}$ are the prescribed vertices, there is no way to use the vertex of the $K_{1}$ in a desired partition if $a_{i} \geq 3$ for all $1 \leq i \leq t$.

In [7], Enomoto and Ota showed positive results in the case $t=3$ or $a_{i}=3$ for all $1 \leq i \leq t$. Later Kawarabayashi [10] showed that if $\sigma_{2}(G) \geq$ $\sum_{i=1}^{t} \max \left\{\left\lfloor\frac{4}{3} a_{i}\right\rfloor, a_{i}+1\right\}-1$, there exist $t$ vertex disjoint paths desired in Conjecture 1. Magnant and Martin [11] proved an asymptotic version of this Conjecture 1 very similar to the results contained in this work but, in general, the conjecture is still unsolved.

The above results and conjecture are concentrated on a partition into paths with one specified end vertex. Along these lines, we have a question; what happens if we specify both end vertices? Egawa et al. [4] showed a result on a partition into cycles containing specified edges, and as a corollary, we obtain the following result.

Theorem 3 (Egawa et al. [4], Enomoto [6]). Let $t$ be an integer with $t \geq 2$ and let $G$ be a graph of order $n \geq 4 t-1$. If $\sigma_{2}(G) \geq n+2 t-2$, then for any $2 t$ vertices $x_{1}, x_{2}, \ldots, x_{t}, y_{1}, \ldots, y_{t}$, there exist $t$ vertex disjoint paths $P_{1}, P_{2}, \ldots, P_{t}$ such that $V(G)=\bigcup_{i=1}^{t} V\left(P_{i}\right)$ and $x_{i}$ and $y_{i}$ are end vertices of $P_{i}$. 
Like Conjecture 1, now we pose a conjecture regarding a partition into paths with both end vertices specified and given order.

Conjecture 2. Let $t$ be an integer with $t \geq 2$ and let $a_{1}, a_{2}, \ldots, a_{t}$ be positive integers. Let $G$ be a graph of order $n=\sum_{i=1}^{t} a_{i}$. If $\sigma_{2}(G) \geq n+2 t-1$, then for any $2 t$ vertices $x_{1}, x_{2}, \ldots, x_{t}, y_{1}, y_{2}, \ldots, y_{t}$, there exist $t$ vertex disjoint paths $P_{1}, P_{2}, \ldots, P_{t}$ such that $V(G)=\bigcup_{i=1}^{t} V\left(P_{i}\right), x_{i}$ and $y_{i}$ are end vertices of $P_{i}$ and $\left|P_{i}\right|=a_{i}$.

The graph $G_{2}=K_{2 t}+\left(K_{1} \cup K_{n-2 t-1}\right)$ shows the sharpness of the $\sigma_{2}(G)$ condition of Conjecture 2. Notice $\sigma_{2}\left(G_{2}\right)=2 t+(n-2 t-2+2 t)=n+2 t-2$. When we specify the $2 t$ vertices from $K_{2 t}$, there is no way to use the vertex of the $K_{1}$ in a desired partition if $a_{i} \geq 4$.

Note that Conjecture 2 is stronger than Conjecture 1. This is because, supposing that Conjecture 2 is true, let $G$ be a graph satisfying the assumption of Conjecture 1 with specified vertices $x_{1}, x_{2}, \ldots, x_{t}$. We construct a new graph $G^{\prime}$ by joining $t$ new vertices $y_{1}, y_{2}, \ldots, y_{t}$ to $G$ and set $a_{i}^{\prime}:=a_{i}+1$ and $n^{\prime}:=n+t$ (then $n^{\prime}$ is the order of $\left.G^{\prime}\right)$. Since $\sigma_{2}\left(G^{\prime}\right) \geq n+t-1+2 t=$ $n^{\prime}+2 t-1$, we can find $t$ vertex disjoint paths $P_{1}^{\prime}, P_{2}^{\prime}, \ldots, P_{t}^{\prime}$ such that $V\left(G^{\prime}\right)=\bigcup_{i=1}^{t} V\left(P_{i}^{\prime}\right), x_{i}$ and $y_{i}$ are end vertices of $P_{i}^{\prime}$ and $\left|P_{i}^{\prime}\right|=a_{i}^{\prime}=a_{i}+1$. Then $t$ disjoint paths $P_{i}:=P_{i}^{\prime}-\left\{y_{i}\right\}$ are desired paths in Conjecture 1 .

Therefore, we know that Conjecture 2 is at least as hard as Conjecture 1. The difficulty of Conjectures 1 and 2 seems to arise from considering a partition into paths with specified end vertex and given "exact" order. In fact, when we allow some flexibility or "tolerance" in the desired order, the situation is different. The main purpose of this paper is to find a partition into paths with both end vertices specified and with given order up to some "tolerance".

Theorem 4. Let $t$ be an integer with $t \geq 2$. For any set of $t$ positive real numbers $\gamma_{1}, \gamma_{2}, \ldots, \gamma_{t}$ with $\sum_{i=1}^{t} \gamma_{i}=1$ and for every $\epsilon>0$ with $\epsilon<\min \left\{\frac{1}{18^{2} t^{2}}, \frac{\gamma_{i}}{2}\right\}$, there exists an integer $n_{0}$ such that for every $(2 t+1)$ connected graph $G$ of order $n \geq n_{0}$ with $\sigma_{2}(G) \geq n+2 t-2$ and for every $2 t$ vertices $x_{1}, x_{2}, \ldots, x_{t}, y_{1}, \ldots, y_{t}$, there exist $t$ vertex disjoint paths $P_{1}, P_{2}, \ldots, P_{t}$ such that $V(G)=\bigcup_{i=1}^{t} V\left(P_{i}\right), x_{i}$ and $y_{i}$ are end vertices of $P_{i}$ and $\left(\gamma_{i}-\epsilon\right) n \leq\left|P_{i}\right| \leq\left(\gamma_{i}+\epsilon\right) n$ for all $1 \leq i \leq t$.

We prove Theorem 4 in Section 4 . Since $\sigma_{2}(G) \geq n+2 t-1$ implies $\kappa(G) \geq 2 t+1$, this result immediately implies the following, which has a slightly larger degree sum condition in place of the connectivity assumption. Theorem 4 is stated and proven as above so it can be used in the proof of Theorem 6 . 
Corollary 5. Let $t$ be an integer with $t \geq 2$. For any set of $t$ positive real numbers $\gamma_{1}, \gamma_{2}, \ldots, \gamma_{t}$ with $\sum_{i=1}^{t} \gamma_{i}=1$ and for every $\epsilon>0$ with $\epsilon<$ $\min \left\{\frac{1}{18^{2} t^{2}}, \frac{\gamma_{i}}{2}\right\}$, there exists an integer $n_{0}$ such that for every graph $G$ of order $n \geq n_{0}$ with $\sigma_{2}(G) \geq n+2 t-1$ and for every $2 t$ vertices $x_{1}, x_{2}, \ldots, x_{t}$, $y_{1}, \ldots, y_{t}$, there exist $t$ vertex disjoint paths $P_{1}, P_{2}, \ldots, P_{t}$ such that $V(G)=$ $\bigcup_{i=1}^{t} V\left(P_{i}\right), x_{i}$ and $y_{i}$ are end vertices of $P_{i}$ and $\left(\gamma_{i}-\epsilon\right) n \leq\left|P_{i}\right| \leq\left(\gamma_{i}+\epsilon\right) n$ for all $1 \leq i \leq t$.

Now we consider a partition into cycles. Posa [15] showed that with large degrees, one can guarantee a 2-factor with a bounded number of cycles. Egawa et al. [4] gave a $\sigma_{2}(G)$ condition to guarantee the existence of a partition into cycles each of which contains one specified vertices. On the other hand, El-Zahar [5] considered a partition into cycles with given order and posed a famous conjecture.

Similarly to a partition into paths, we pose the following conjecture. Moreover, in this paper, we also show a result on such a partition with some "tolerance".

Conjecture 3. Let $t$ be an integer with $t \geq 2$ and let $a_{1}, a_{2}, \ldots, a_{t}$ be positive integers. Let $G$ be a graph of order $n=\sum_{i=1}^{t} a_{i}$. If $\sigma_{2}(G) \geq n+2 t-2$, then for any $t$ vertices $x_{1}, x_{2}, \ldots, x_{t}$, there exist $t$ vertex disjoint cycles $C_{1}, C_{2}, \ldots, C_{t}$ such that $V(G)=\bigcup_{i=1}^{t} V\left(C_{i}\right), x_{i} \in V\left(C_{i}\right)$ and $\left|C_{i}\right|=a_{i}$ for all $1 \leq i \leq t$.

Theorem 6. Let $t$ be an integer with $t \geq 2$. For any set of $t$ positive real numbers $\gamma_{1}, \gamma_{2}, \ldots, \gamma_{t}$ with $\sum_{i=1}^{t} \gamma_{i}=1$ and for every $\epsilon>0$ with $\epsilon<\min \left\{\frac{1}{18^{2} t^{2}}, \frac{\gamma_{i}}{2}\right\}$, there exists an integer $n_{0}$ such that for every graph $G$ of order $n \geq n_{0}$ with $\sigma_{2}(G) \geq n+2 t-2$ and for every $t$ vertices $x_{1}, x_{2}, \ldots, x_{t}$, there exist $t$ vertex disjoint cycles $C_{1}, C_{2}, \ldots, C_{t}$ such that $V(G)=\bigcup_{i=1}^{t} V\left(C_{i}\right), x_{i} \in V\left(C_{i}\right)$ and $\left(\gamma_{i}-\epsilon\right) n \leq\left|C_{i}\right| \leq\left(\gamma_{i}+\epsilon\right) n$ for all $1 \leq i \leq t$.

The sharpness of Conjecture 3 is given by $G_{3}=K_{2 t-1}+\left(K_{t} \cup K_{n-3 t+1}\right)$. Notice $\sigma_{2}\left(G_{2}\right)=(t-1+2 t-1)+(n-3 t+2 t-1)=n+2 t-3$. If the vertices of the $K_{t}$ are the prescribed vertices, there is no way to construct $t$ vertex disjoint cycles each of which containing exactly one prescribed vertex regardless of the choice of $a_{i}$. The proof of Theorem 6 is also left to Section 4 .

Denote the distance, along a path $P$, between vertices $u$ and $v$ by $\operatorname{dist}_{P}(u, v)$. All standard notation can be found in [1].

\section{Preliminary results}

Along with the classical results of Ore [13], Menger [12] and Dirac [3], the proofs of Theorems 4 and 6 use the following results of Williamson [17], 
Ore [14] and Thomas and Wollan [16]. A graph is said to be panconnected (or hamilton-connected) if, between any pair of vertices $x, y \in V(G)$, there exists a path of every length $l$ for $d \leq l \leq|G|-1$ (a path of length $|G|-1$, respectively), where $d$ is the length of a shortest path connecting $x$ and $y$. Williamson [17], and Ore [14] proved the following sufficient conditions for a graph to be panconnected, and hamilton-connected, respectively.

Theorem 7 (Williamson [17]). Given a graph $G$ of order $n$, if $\delta(G) \geq \frac{n+2}{2}$ then $G$ is panconnected.

Theorem 8 (Ore [14]). Given a graph $G$ of order $n$, if $\sigma_{2}(G) \geq n+1$ then $G$ is hamilton-connected.

A graph is said to be $t$-linked if, for every choice of $2 t$ vertices $x_{1}, \ldots, x_{t}$ and $y_{1}, \ldots, y_{t}$, there exists $t$ vertex disjoint paths $P_{i}$ such that $x_{i}$ and $y_{i}$ are end vertices of $P_{i}$ for all $i$. We use the following result.

Theorem 9 (Thomas and Wollan [16]). If a graph $G$ is $10 t$-connected, then $G$ is t-linked.

Our proof includes three main steps. The first creates a spanning collection of vertex disjoint paths (or cycles) starting at the chosen vertices. The second step moves vertices from paths which are long enough to paths which are too short if certain conditions are satisfied. When these conditions are not satisfied, we prove the graph has strong structure which allows us, in the third step, to build the desired path (or cycle) system directly.

\section{Lemmas}

Now we consider only a collection of paths. Theorem 4 will be used to prove Theorem 6 so there is no need to consider lemmas explicitly for Theorem 6 .

For the sake of notation, we define some terminology. Throughout this and the next section, suppose we are given an integer $t \geq 3, t$ positive real numbers $\gamma_{1}, \ldots, \gamma_{t}$ with $\sum \gamma_{i}=1$ and a real number $0<\epsilon \leq \frac{1}{18^{2} t^{2}}$ such that $\epsilon \leq \frac{\gamma_{i}}{2}$ for all $1 \leq i \leq t$. Let $G$ be a $(2 t+1)$-connected graph of order $n$ sufficiently large (compared to $t, \gamma_{i}$ 's and $\epsilon$ ) with a set $S=$ $\left\{x_{1}, \ldots, x_{t}, y_{1}, \ldots, y_{t}\right\} \subset V(G)$ of $2 t$ prescribed vertices. A collection of vertex disjoint paths $\mathscr{P}=\left\{P_{1}, P_{2}, \ldots, P_{t}\right\}$ is called a path collection for $S$ if $P_{i}$ connects $x_{i}$ and $y_{i}$ and $\left|P_{i}\right| \geq \epsilon n$ for any $1 \leq i \leq t$. A path collection $\mathscr{P}$ for $S$ is spanning if $\bigcup_{i=1}^{t} V\left(P_{i}\right)=V(G)$ and is desired if $\left(\gamma_{i}-\epsilon\right) n<\left|P_{i}\right|<\left(\gamma_{i}+\epsilon\right) n$ for all $1 \leq i \leq t$.

Since there are many small constants used in this work, to simplify computations, we will frequently assume the constants, the value of $n$ and 
other variables used are carefully chosen to satisfy divisibility. For example, in place of $\lceil\epsilon n\rceil$, we will occasionally treat the quantity $\epsilon n$ as an integer.

Our first lemma allows us to absorb vertices into path collections. In this process, we must allow some paths to get shorter. We carefully allow only very little decrease in length and only from paths which are long enough to sustain such a loss. The goal of the following lemma is to allow only paths of length at least $2 \epsilon n$ to get shorter and only by a limited amount.

Lemma 1 (Absorbing). Let $\mathscr{P}=\left\{P_{1}, P_{2}, \ldots, P_{t}\right\}$ be a path collection for $S$. If $\sigma_{2}(G) \geq n+2 t-2, \kappa(G) \geq 2 t+1$ and $n>\frac{20 t}{\epsilon}$, then there exists a spanning path collection for $S, \mathscr{P}^{\prime}=\left\{P_{1}^{\prime}, P_{2}^{\prime}, \ldots, P_{t}^{\prime}\right\}$ such that $\left|P_{i}^{\prime}\right| \geq\left|P_{i}\right|$ if $\left|P_{i}\right|<2 \epsilon n$ and $\sum_{j=1}^{t} \max \left\{\left|P_{j}\right|-\left|P_{j}^{\prime}\right|, 0\right\} \leq \frac{20}{\epsilon^{2}}$.

Proof. Let $G$ be a graph as given above, let $P=\bigcup_{i=1}^{t} V\left(P_{i}\right)$ and let $J=$ $V(G) \backslash P$. We suppose $\mathscr{P}$ is the largest such path collection or, in other words, suppose $|J|$ is minimum under the assumption each path is not shorter than its corresponding original. The following claims provide structure with which we prove the desired result.

Claim 1. For every vertex $v \in J, d_{P}(v) \leq \frac{|P|+2 t}{3}$.

Proof of Claim 1. Let $v \in J$. The vertex $v$ cannot be adjacent to any pairs of vertices $u$ and $u^{+}$which are consecutive along a path $P_{i}$ as $P_{i}$ could then be replaced with $P_{i}^{\prime}=\ldots, u, v, u^{+}, \ldots$ to form a longer path collection and contradict the assumptions. This observation implies that $d_{P}(v) \leq \frac{|P|+t}{2}$, which means $d(v) \leq \frac{|P|+t}{2}+|J|-1$. Suppose $d_{P}(v)>\frac{|P|+2 t}{3}$. By the pigeonhole principle, there exists a vertex $u \in P_{i}$ for some $1 \leq i \leq t$ with $v u^{+}, v u^{-} \in E(G)$. By the above observation, we know $u v \notin E(G)$ and we may also assume $N_{J}(u) \cap N_{J}(v)=\emptyset$. So $d(v)+d(u) \geq n+2 t-2$. Since $d_{P}(v) \leq \frac{|P|+t}{2}$ and $d_{J}(v)+d_{J}(u) \leq|J|-1$, we get

$$
\begin{aligned}
d_{P}(u) & \geq n+2 t-2-d(v)-d_{J}(u) \\
& \geq n+2 t-\frac{|P|+t}{2}-|J|-1 \\
& =\frac{|P|}{2}+\frac{3 t}{2}-1 .
\end{aligned}
$$

This implies that $u$ must be adjacent to two consecutive vertices along some path $P_{j} \in \mathscr{P}$. Therefore, we may absorb $u$ into $P_{j}$ as above and replace $P_{i}$ with $P_{i}^{\prime}=\ldots, u^{-}, v, u^{+}, \ldots$ to form a larger path collection, contradicting our original assumption. 
Claim 2. The graph induced on $J$ is hamilton-connected.

Proof of Claim 2. Suppose there exist vertices $x, y \in J$ with $x y \notin E(G)$. By the assumed degree condition, $d_{G}(x)+d_{G}(y) \geq n+2 t-2$ but by Claim 1 , $d_{P}(x), d_{P}(y) \leq \frac{|P|+2 t}{3}$. Therefore

$$
\begin{aligned}
d_{J}(x)+d_{J}(y) & \geq n+2 t-2-2\left(\frac{|P|+2 t}{3}\right) \\
& =|J|+\frac{|P|+2 t}{3}-2 \\
& \geq|J|+2 .
\end{aligned}
$$

By Theorem $8, J$ is hamilton-connected.

$\square_{\text {Claim } 2}$

Claim 3. $\left|\bigcup_{v \in J} N_{P}(v)\right| \geq 2 t+1$. In particular, there exists a path $P_{i}$ such that $\left|\bigcup_{v \in J} N_{P_{i}}(v)\right| \geq 3$.

Proof of Claim 3. Since $G$ is $(2 t+1)$-connected, the first statement is obvious. The second statement of Claim 3 directly follows from the fact that $\left|\bigcup_{v \in J} N_{P}(v)\right| \geq 2 t+1$ and the pigeonhole principle.

$\square_{\text {Claim } 3}$

Let $J_{P}=\cup_{j \in J} N_{P}(j)$ be the set of all neighbors in $P$ of vertices of $J$.

Claim 4. For every vertex $v \in J, d_{P}(v) \geq \frac{\epsilon}{4} n$.

Proof of Claim 4. Suppose that there exists a vertex $v \in J$ such that $d_{P}(v)<$ $\frac{\epsilon}{4} n$. Let $A$ be a shortest path segment of $P$ (not including end vertices) between vertices in $J_{P}$ and let $P_{i}$ be the path containing $A$. Note that $|A|<\frac{\left|P_{i}\right|}{2}$, by Claim 3. The goal of this claim is to move the vertices of $A$ into $P_{i} \backslash A$ and absorb $v$ into $P_{i}$ as above, which contradicts the minimality of $|J|$.

Let $w$ be any vertex of $A$. The segment $A$ was chosen to be the smallest between edges from $J$ so $w x \notin E(G)$ for all $x \in J$ and, in particular, $w v \notin E(G)$. Therefore we know

$$
\begin{aligned}
d_{P}(w) & \geq n+2 t-2-d(v) \\
& >n-|J|-\frac{\epsilon}{4} n+2 t-1 \\
& \geq|P|-\frac{\left|P_{i}\right|}{4}+2 t-1 \\
& \geq\left(|P|-\left|P_{i}\right|\right)+\frac{3\left|P_{i}\right|}{4}+2 t-1
\end{aligned}
$$




$$
\begin{aligned}
& >\left(|P|-\left|P_{i}\right|\right)+\frac{|A|}{2}+\frac{\left|P_{i}\right|}{2}+2 t-1 \\
& =\left(|P|-\left|P_{i}\right|\right)+|A|+\frac{\left|P_{i}\right|-|A|}{2}+2 t-1 .
\end{aligned}
$$

This implies that for each $w \in A$, there exists a pair of consecutive vertices $u, u^{+} \in P_{i} \backslash A$ such that $w u, w u^{+} \in E(G)$ so we may move the vertices of $A$, one at a time in order to reapply the above argument, into $P_{i} \backslash A$ and absorb at least one vertex of $J$ (or possibly all of $J$ ) into $P_{i}$.

$\square$ Claim 4

Claim 5. $|J|<\frac{5}{\epsilon}$.

Proof of Claim 5. Suppose $|J| \geq \frac{5}{\epsilon}$. Let $\ell$ be the number of path segments between vertex disjoint edges from $J$ to $P$. Since $J$ is hamilton-connected (by Claim 2), we know these segments must have length at least $|J|+1$. Let $L$ be the set of vertices in the segments above including the endvertices. Thus $|L| \geq \ell(|J|+1)$ but furthermore, $d_{L}(j) \leq \ell+t$ for all $j \in J$ so $L$ has at most $(\ell+t)|J|$ edges to $J$. Also note that every vertex of $P$ with at least 2 edges to $J$ must be in $L$. This means that $P \backslash L$ has at most $|P \backslash L|$ edges to $J$. Conversely, by Claim $4, d_{P}(j) \geq \frac{\epsilon n}{4}$ for all $j \in J$ meaning that there are at least $\frac{|J| \epsilon n}{4}$ edges between $P$ and $J$. This implies

$$
(\ell+t)|J|+|P \backslash L| \geq \frac{\epsilon n|J|}{4}
$$

or, since $|J| \geq \frac{5}{\epsilon}$

$$
|P \backslash L| \geq \frac{5 n}{4}-\frac{5(\ell+t)}{\epsilon} .
$$

With $|L| \geq \ell(|J|+1)>\frac{5 \ell}{\epsilon}$ and $|P|<n$, this implies that $\frac{n}{4}<\frac{5 t}{\epsilon}$, a contradiction.

By Claims 4 and 5, we may assume $d_{P}(v) \geq \frac{\epsilon n}{4}$ for any $v \in J$ and $|J|<\frac{5}{\epsilon}$. The rest of the proof is similar to the proof of Claim 4. We again consider a segment $A$ between adjacencies of a vertex in $J$. Unfortunately, this time we have to be more careful about how we remove the vertices of $A$ from the path.

Let $J^{\prime}:=\left\{v \in J: d_{P}(v) \geq \frac{n}{2 t+1}\right\}$ and let $\bar{J}:=J-J^{\prime}$. First, we will absorb a vertex in $J^{\prime}$ into paths in $\mathscr{Q}$, where $\mathscr{Q}:=\left\{P_{i} \in \mathscr{P}:\left|P_{i}\right| \geq 2 \epsilon n\right\}$. Note that $\mathscr{Q} \neq \emptyset$. Let $\mathscr{Q}=\left\{Q_{1}, Q_{2}, \ldots\right\}$ and let $Q$ be the set of vertices in paths of $\mathscr{Q}$. There are only $t$ total paths, so we know $|Q| \geq(1-2(t-1) \epsilon) n-|J|$ 
and, since $n$ is sufficiently large and $|J|<\frac{5}{\epsilon}$, we get $|Q| \geq(1-3 t \epsilon) n$. Since $\epsilon$ was chosen such that $\epsilon \leq \frac{1}{6 t(2 t+1)}$, we know that $|Q| \geq\left(1-\frac{1}{2(2 t+1)}\right) n$.

Let $v \in J^{\prime}$. Since $|P \backslash Q| \leq \frac{n}{2(2 t+1)}$ and $d_{P}(v) \geq \frac{n}{2 t+1}$, we have $d_{Q}(v) \geq$ $\frac{n}{2(2 t+1)}$. Let $A$ be the smallest segment of a path $Q_{i} \in \mathscr{Q}$ between (not including end vertices) two vertices adjacent to $v$. Since $d_{Q}(v) \geq \frac{n}{2(2 t+1)}$, we may choose $n$ sufficiently large such that

$$
|A| \leq \frac{\left(1-\frac{1}{2(2 t+1)}\right) n}{\frac{n}{2(2 t+1)}-t} \leq 3(2 t+1) .
$$

Let $w \in A$. Of course $v w \notin E(G)$ so by Claim 1 ,

$$
\begin{aligned}
d_{P}(w) & \geq n+2 t-2-\left(\frac{|P|+2 t}{3}+|J|-1\right)-(|J|-1) \\
& =\frac{|P|+t+|A|}{2}+\frac{|P|}{6}+\frac{13 t}{6}-\frac{|A|}{2}-|J| \\
& \geq \frac{|P|+t+|A|}{2},
\end{aligned}
$$

and we may absorb $w$ into $P \backslash A$. This same process may be repeated for all $w \in A$ and we may then absorb $v$ into $Q$. By this process, the path $Q_{i}$ loses at most $|A| \leq 3(2 t+1)$ vertices. We repeat this process for each vertex of $J^{\prime}$. This means that paths of $\mathscr{Q}$ lose at most a total of $\left|J^{\prime}\right|(6 t+3)$ vertices.

Note that we are now left with $d_{P}(v)<\frac{n}{2 t+1}$ for all $v \in \bar{J}$. Let $v \in \bar{J}$ and choose a smallest segment $A$ along a path $P_{i}$ between two adjacencies of $v$. Since $d_{P}(v) \geq \frac{\epsilon n}{4}$, we know the length of this segment is at most $|A| \leq \frac{n}{\frac{\epsilon}{4}-t}<\frac{5}{\epsilon}$ for $n$ sufficiently large. If $\left|P_{i}\right| \geq 2 \epsilon n$, we absorb $v$ as above. This creates a new path $P_{i}^{\prime}$ with $\left|P_{i}^{\prime}\right| \geq\left|P_{i}\right|-\frac{5}{\epsilon}$. Therefore we suppose $A$ is a subpath of a path $P_{j}$ where $\epsilon n \leq\left|P_{j}\right|<2 \epsilon n$.

Let $u$ be a vertex of $A$ and note that, since $A$ was chosen to be the smallest such segment, $u v \notin E(G)$. This means that

$$
\begin{aligned}
d(u) & \geq n+2 t-2-d(v) \\
& >n+2 t-2-\left(\frac{n}{2 t+1}+|J|-1\right) \\
& \geq\left(1-\frac{1}{2 t}\right) n \\
& \geq \frac{5 n}{6}
\end{aligned}
$$




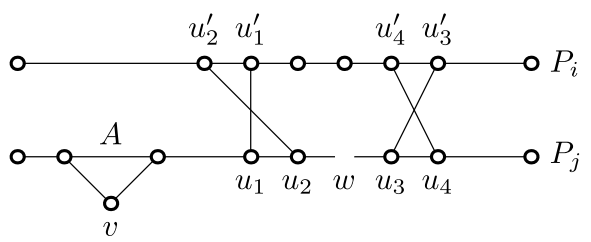

Figure 1: Rearrangement of paths.

since $n$ is sufficiently large and $t \geq 3$. Certainly the same is true for every vertex $u \in V\left(P_{j}\right) \backslash N(v)$. Assign an ordering $\ell$ to the vertices of each path $P_{i}$ such that $\ell(u)=\operatorname{dist}_{P_{i}}\left(x_{i}, u\right)+1$. Since $v$ cannot be adjacent to two vertices of $P_{j}$ with only one vertex in between, there exist four distinct vertices $u_{1}, u_{2}, u_{3}, u_{4} \in P_{j} \backslash(A \cup N(v))$ such that $\ell\left(u_{1}\right)=\ell\left(u_{2}\right)-1, \ell\left(u_{3}\right)=\ell\left(u_{4}\right)-1$ and $\ell\left(u_{2}\right)+1 \leq \ell\left(u_{3}\right) \leq \ell\left(u_{2}\right)+2$.

Let $p=|P \backslash Q|$ and suppose $\frac{5 n}{6}-p<\frac{19}{24}(n-p-|J|)$. Since $p \leq 2 t \epsilon n$, this inequality implies $n<10 t \epsilon n-19|J|$, contradicting the fact that $n$ is sufficiently large. Thus, $d_{Q}\left(u_{i}\right) \geq \frac{5 n}{6}-p \geq \frac{19}{24}(n-|J|-p)=\frac{19}{24}|Q|$ for each vertex $u_{i}$. By the pigeonhole principle, there exists at least $2 \cdot \frac{19}{24}|Q|-t-|Q|=$ $\frac{7}{12}|Q|-t$ pairs of distinct vertices $u_{1}^{\prime}, u_{2}^{\prime}$ ( similarly $\left.u_{3}^{\prime}, u_{4}^{\prime}\right)$ in paths of $\mathscr{Q}$ with $u_{i} u_{i}^{\prime} \in E(G)$ for all $i$ and $\ell\left(u_{1}^{\prime}\right)=\ell\left(u_{2}^{\prime}\right)+1$ (similarly $\left.\ell\left(u_{3}^{\prime}\right)=\ell\left(u_{4}^{\prime}\right)+1\right)$.

Since $2\left(\frac{7}{12}|Q|-t\right)-|Q|-3 t>0$, we may again apply the pigeonhole principle. Hence, there exists a set of four vertices $u_{1}^{\prime}, u_{2}^{\prime}, u_{3}^{\prime}, u_{4}^{\prime}$ in a single path $P_{i} \in \mathscr{Q}$ such that $\ell\left(u_{1}^{\prime}\right)=\ell\left(u_{2}^{\prime}\right)+1, \ell\left(u_{3}^{\prime}\right)=\ell\left(u_{4}^{\prime}\right)+1$ and $\ell\left(u_{4}^{\prime}\right)=$ $\ell\left(u_{1}^{\prime}\right)+3$. See Figure 1 where the shaded vertex $w$ may or may not be present.

At this point we may swap $P_{j}=\ldots, u_{1}, u_{2}, \ldots, u_{3}, u_{4}, \ldots$ for $P_{j}^{\prime}=\ldots$, $u_{1}, u_{1}^{\prime}, \ldots, u_{4}^{\prime}, u_{4}, \ldots$ and $\operatorname{swap} P_{i}=\ldots, u_{2}^{\prime}, u_{1}^{\prime}, \ldots, u_{4}^{\prime}, u_{3}^{\prime}, \ldots$ for $P_{i}^{\prime}=\ldots$, $u_{2}^{\prime}, u_{2}, \ldots, u_{3}, u_{3}^{\prime}, \ldots$ This swap makes $\left|P_{j}\right|+1 \leq\left|P_{j}^{\prime}\right| \leq\left|P_{j}\right|+2$ and $\left|P_{i}\right|-2 \leq$ $\left|P_{i}^{\prime}\right| \leq\left|P_{i}\right|-1$. The swap process may be repeated as necessary to make $\left|P_{j}^{\prime}\right| \geq\left|P_{j}\right|+|A|$ (thereby removing at most $|A|+1<\frac{5}{\epsilon}+1$ vertices from paths of $\mathscr{Q})$.

We may now absorb $v$ into $P_{j}^{\prime}$, losing the vertices of $A$ to other paths as above. Again, we may repeat this process to absorb all of $\bar{J}$. In both processes, we lose at most a total of $\left|J^{\prime}\right|(6 t+3)+|\bar{J}|\left(\frac{5}{\epsilon}+1-1\right) \leq|J| \frac{5}{\epsilon}$ vertices from $\mathscr{Q}$. Thus, for all $P_{j} \in \mathscr{Q}$, we have $\left|P_{j}^{\prime}\right| \geq 2 \epsilon n-\frac{5}{\epsilon} \cdot \frac{5}{\epsilon} \geq \epsilon n$. Moreover, we also know that $\sum_{i=1}^{t} \max \left\{\left|P_{i}\right|-\left|P_{i}^{\prime}\right|, 0\right\} \leq \frac{20}{\epsilon^{2}}$. This completes the proof of Lemma 1.

Our next lemma requires some specific definitions. Suppose we are given a path collection $\mathscr{P}=\left\{P_{1}, \ldots, P_{t}\right\}$. Let $f\left(P_{i}\right)=\gamma_{i} n-\left|P_{i}\right|$. Without loss of 
generality, suppose the paths $P_{i}$ are ordered such that $f\left(P_{i}\right) \geq f\left(P_{i+1}\right)$. By the definition of $f\left(P_{i}\right)$ and $\gamma_{i}$ 's, note that $f\left(P_{1}\right) \geq 0$, because otherwise,

$$
\begin{aligned}
0 & >\sum_{i=1}^{t} f\left(P_{i}\right) \\
& =\sum_{i=1}^{t} \gamma_{i} n-\sum_{i=1}^{t}\left|P_{i}\right| \\
& \geq n-n=0,
\end{aligned}
$$

a contradiction. Define:

$$
\mu(\mathscr{P})=\sum_{i=1}^{t}(t+1-i) f\left(P_{i}\right) .
$$

Proposition 1. If $\mathscr{P}$ is a spanning path collection but not desired, then there exists an integer $k$ such that $f\left(P_{k}\right)-f\left(P_{k+1}\right)>\frac{\epsilon}{t^{2}} n$ and $f\left(P_{k+1}\right)<$ $-\frac{\epsilon}{t^{2}} n$.

Proof. If $f\left(P_{t}\right)>-\frac{\epsilon}{t} n$, we get $\left|P_{i}\right|<\left(\gamma_{i}+\frac{\epsilon}{t}\right) n<\left(\gamma_{i}+\epsilon\right) n$ for all $i$, which implies $\left|P_{i}\right|=n-\sum_{j \neq i}\left|P_{j}\right|>\left(\gamma_{i}-\epsilon\right) n$ for all $i$. This contradicts the assumption $\mathscr{P}$ is not a desired path collection. Thus, $f\left(P_{t}\right) \leq-\frac{\epsilon}{t} n$.

Suppose that $f\left(P_{k}\right)-f\left(P_{k+1}\right) \leq \frac{\epsilon}{t^{2}} n$ for all $1 \leq k \leq t-1$. Then $f\left(P_{1}\right) \leq f\left(P_{t}\right)+(t-1) \frac{\epsilon}{t^{2}} n<\frac{-\epsilon}{t^{2}} n<0$ because $f\left(P_{t}\right) \leq-\frac{\epsilon}{t} n$, a contradiction. Therefore there exists an integer $k$ such that $f\left(P_{k}\right)-f\left(P_{k+1}\right)>\frac{\epsilon}{t^{2}} n$. If we take such an integer $k$ as large as possible, we have $f\left(P_{k+1}\right)<-\frac{\epsilon}{t^{2}} n$ because $f\left(P_{t}\right)<-\frac{\epsilon}{t} n$ and $f\left(P_{i}\right)-f\left(P_{i+1}\right) \leq \frac{\epsilon}{t^{2}} n$ for any $k+1 \leq i \leq t-1$.

Next we extract a lemma from [8] in a form that is appropriate to our current situation. This lemma takes two paths and, under certain conditions, shows the existence of two other paths which are, in some sense, better. For ease of notation, let $c=\frac{\epsilon^{2}}{24 \cdot 48 t^{2}}$.

Lemma 2 (Swapping). Let $\mathscr{P}=\left\{P_{1}, P_{2}, \ldots, P_{t}\right\}$ be a spanning path collection for $S$ but not desired. If $\kappa(G) \geq 2 t+1, \sigma_{2}(G) \geq n+2 t-2$ and if $e(\mathscr{A}, \mathscr{P}-\mathscr{A}) \geq c t^{2} n^{2}$ for any subcollection $\mathscr{A}$ of $\mathscr{P}$, then there exists a spanning path collection $\mathscr{Q}$ such that $\mu(\mathscr{Q})<\mu(\mathscr{P})$.

Proof. Let $\mathscr{P}$ be as stated. By Proposition 1, there exists an integer $k$ with $f\left(P_{k}\right)-f\left(P_{k+1}\right) \geq \frac{\epsilon}{t^{2}} n$ and $f\left(P_{k+1}\right)<\frac{-\epsilon}{t^{2}} n$. Let $\mathscr{A}=\left\{P_{i}: 1 \leq i \leq k\right\}$. Since $e(\mathscr{A}, \mathscr{P}-\mathscr{A}) \geq c t^{2} n^{2}$, there must exist paths $P_{i} \in \mathscr{A}$ and $P_{j} \in \mathscr{P}-\mathscr{A}$ with $e\left(P_{i}, P_{j}\right) \geq c n^{2}$. Notice that $1 \leq i \leq k$ and $k+1 \leq j \leq t$. 


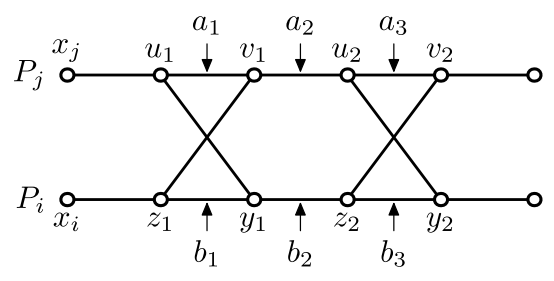

Figure 2: Swapping.

Let $A=V\left(P_{j}\right)$ and $B=V\left(P_{i}\right)$. Let $A^{\prime} \subseteq A$ denote the set of vertices $v \in A$ with $d_{B}(v) \geq \frac{c n^{2}}{2|A|}$. Since $|A| \cdot|B| \leq \frac{n^{2}}{4}$, note that $d_{B}(v) \geq \frac{c n^{2}}{2|A|} \geq 2 c|B|$ for any $v \in A^{\prime}$. Since $e(A, B) \geq c n^{2}$, we find

$$
\begin{aligned}
\left|A^{\prime}\right| & \geq \frac{c n^{2}-\left(|A|-\left|A^{\prime}\right|\right)\left(\frac{c n^{2}}{2|A|}\right)}{|B|} \\
& \geq \frac{c n^{2}}{2|B|} \geq 2 c|A| .
\end{aligned}
$$

Assign a labeling $l(v)$ of the vertices of $A$ and $B$ given by the distance along $P_{j}$ or $P_{i}$ from $x_{j}$ or $x_{i}$ respectively. Define a crossing pair to be a pair of edges $u y$ and $v z$ with $u, v \in A$ and $y, z \in B$ such that $l(u)<l(v)$ and $l(z)<l(y)$. Define the gap of a crossing pair to be $|l(y)-l(z)|-1$. We will concern ourselves only with crossing pairs with gap length at most $\frac{4}{c}$.

Consider Figure 2 consisting of two crossing pairs $u_{1} y_{1}, v_{1} z_{1}$ and $u_{2} y_{2}$, $v_{2} z_{2}$ where $a_{1}=l\left(v_{1}\right)-l\left(u_{1}\right)-1 \geq 0, a_{2}=l\left(u_{2}\right)-l\left(v_{1}\right)+1>0, a_{3}=$ $l\left(v_{2}\right)-l\left(u_{2}\right)-1>0, b_{1}=l\left(y_{1}\right)-l\left(z_{1}\right)-1>0, b_{2}=l\left(z_{2}\right)-l\left(y_{1}\right)+1 \geq 0$ and $b_{3}=l\left(y_{2}\right)-l\left(z_{2}\right)-1>0$. The goal of this lemma is to create new paths $P_{j}^{\prime}=$ $x_{j}, \ldots, u_{1}, y_{1}, \ldots, z_{2}, v_{2}, \ldots, y_{j}$ and $P_{i}^{\prime}=x_{i}, \ldots, z_{1}, v_{1}, \ldots, u_{2}, y_{2}, \ldots, y_{i}$ and by using Lemma 1 to absorb vertices which are removed by this operation. Note that $\left|P_{j}^{\prime}\right|=\left|P_{j}\right|-\left(a_{1}+a_{2}+a_{3}\right)+b_{2}$ and $\left|P_{i}^{\prime}\right|=\left|P_{i}\right|-\left(b_{1}+b_{2}+b_{3}\right)+a_{2}$.

First, we find two crossing pairs as in Figure 2 which satisfy:

$$
\begin{aligned}
a_{2}-b_{2} & \geq \frac{64 t+112}{c^{2}}, \\
a_{1}+a_{3}+b_{1}+b_{3} & \leq \frac{32+8 c}{c^{2}} \\
a_{1}+a_{2}+a_{3}-b_{2} & <\frac{\epsilon}{2 t^{2}} n \\
a_{2}-\left(b_{1}+b_{2}+b_{3}\right) & <\frac{\epsilon}{2 t^{2}} n
\end{aligned}
$$


Partition the vertices of $A^{\prime}$ into collections of $\left\lceil\frac{4}{3 c}\right\rceil$ consecutive (within $\left.A^{\prime}\right)$ vertices. As mentioned earlier, we will assume constants are chosen so divisibility is satisfied so, as opposed to sticking with $\left\lceil\frac{4}{3 c}\right\rceil$, we will simply write $\frac{4}{3 c}$. Call each such collection a chunk.

Claim 6. Given a chunk $C$, there are at least $\frac{c|B|}{2}$ crossing pairs within $C$, all vertex disjoint in $B$ with gap length at most $\frac{4}{c}$.

Proof of Claim 6. We will find many desired crossing pairs iteratively. Suppose we already found $r$ crossing pairs. In other words, $2 r$ vertices of $B$ are used for the $r$ crossing pairs and we cannot use such $2 r$ vertices for crossing pairs we will find after this. If $r \geq \frac{c|B|}{2}$, there is nothing to prove. So we may assume that $r<\frac{c|B|}{2}$. Suppose that there exists no more crossing pair with gap length at most $\frac{4}{c}$. Label the vertices of the chunk $C$ as $v_{1}, v_{2}, \ldots$ such that $l\left(v_{g}\right)<l\left(v_{h}\right)$ for all $g<h$. We know $d_{B}\left(v_{1}\right) \geq 2 c|B|$ but at most $2 r$ vertices of them are already used for the previous crossing pairs. Therefore there are at least $2 c|B|-2 r \geq c|B|$ new adjacencies of $v_{1}$.

Now suppose we have considered $v_{1}, v_{2}, \ldots, v_{h-1}$ and let $v_{h}$ be the leftmost remaining vertex of $C$ which has not yet been considered. Suppose that $\frac{4}{c}$ consecutive vertices of $B$ have at least two vertices, say $y$ and $z$ with $l(y)>l(z)$, which are not used for the previous crossing pairs and which are shared by $v_{h}$ and one of $v_{1}, v_{2}, \ldots, v_{h-1}$ as neighbors. Then we can find two edges $v_{g} y$ and $v_{h} z$ for some $1 \leq g \leq h-1$, which is a new crossing pair. Therefore $v_{h}$ has at least $2 c|B|-2 r-|B| / \frac{4}{c}>\frac{3 c|B|}{4}$ new adjacencies. This implies that each vertex $v_{h}$ of $C$ forces at least $\frac{3 c|B|}{4}$ new vertices of $B$ not to have any adjacencies in $C \backslash\left\{v_{1}, v_{2}, \ldots, v_{h-1}\right\}$.

After considering $\frac{4}{3 c}$ vertices of $C$, at least

$$
c|B|+\left(\frac{4}{3 c}-1\right) \frac{3 c|B|}{4}=\left(1+\frac{c}{4}\right)|B|
$$

vertices of $B$ are used as adjacencies of $v_{1}, v_{2}, \ldots, v_{\frac{4}{3 c}}$, which is a contradiction and completes the proof of the claim.

$\square_{\text {Claim } 6}$

Given two crossing pairs $u_{1} y_{1}, v_{1} z_{1}$ and $u_{2} y_{2}, v_{2} z_{2}$, we say these pairs form a swapping structure if $l\left(u_{i}\right)>l\left(v_{j}\right)$ and $l\left(z_{i}\right)>l\left(y_{j}\right)$ for some choice of $i, j \in\{1,2\}$. For this choice of $i$ and $j$, define the distance of the swapping structure to be $l\left(z_{i}\right)-l\left(y_{j}\right)+1$ (or the distance in $B$ between the vertices of the crossing pairs).

Claim 7. Within any collection of $\frac{4}{c}$ chunks, there exists a swapping structure with distance $h$ such that $0 \leq h \leq \frac{16}{c^{2}}$. 
Proof of Claim 7. This claim employs a proof almost identical to that of Claim 6. Label the chunks as $C_{1}, C_{2}, \ldots$ in the order of $l$. We first consider the left-most chunk $C_{1}$. This chunk has at least $\frac{c|B|}{2}$ crossing pairs so there are at least $\frac{c|B|}{2}$ right-vertices of these pairs.

As before, we suppose $h-1$ chunks $C_{1}, C_{2}, \ldots, C_{h-1}$ have been considered and consider the left-most chunk $C_{h}$ of the remaining set of chunks. For every $\frac{16}{c^{2}}$ consecutive vertices of $B$, suppose that $C_{h}$ shares at least $\frac{4}{c}$ right-vertices of crossing pairs with $C_{1}, C_{2}, \ldots, C_{h-1}$. Then, there exist two right-vertices $y_{1}$ and $y_{2}$ which are shared by $C_{h}$ and some chunk of $C_{1}, C_{2}, \ldots, C_{h-1}$ such that $l\left(y_{2}\right)-l\left(y_{1}\right) \geq \frac{4}{c}-1$. Let $z_{2}$ be a left-vertex of the crossing pair in $C_{h}$ which corresponds to $y_{2}$. Since the gap of this crossing pair is at most $\frac{4}{c}$, so $l\left(z_{2}\right) \geq l\left(y_{2}\right)-\frac{4}{c}+1 \geq l\left(y_{1}\right)$. Thus, the crossing pair of $C_{h}$ containing $y_{2}$ and $z_{2}$ and the one of $C_{g}$ containing $y_{1}$ for some $1 \leq g \leq h-1$ create a swapping structure with distance at most $\frac{16}{c^{2}}$. Therefore this chunk may share a total of at most $\left(\frac{4}{c}\right)\left(\frac{|B|}{16 / c^{2}}\right)=\frac{c|B|}{4}$ right-vertices with preceding chunks. This implies that at least $\frac{c|B|}{4}$ right-vertices are new.

Hence, after consideration of the $\frac{4}{c}$ chunks, there are

$$
|B|-\frac{c|B|}{2}-\left(\frac{4}{c}-1\right) \frac{c|B|}{4}=\frac{-c|B|}{4}<0
$$

vertices available in $B$ for right-vertices of crossing pairs which is again a contradiction completing the proof of the claim.

$\square_{\text {Claim } 7}$

Given a chunk $C$, define the span of $C$ to be the number of vertices of $A$ between the left-most and right-most vertex of the chunk. Since the chunks have $\frac{4}{3 c}$ vertices of $A^{\prime}$ and $\left|A^{\prime}\right| \geq 2 c|A|$, the total number of chunks is $\left|A^{\prime}\right| / \frac{4}{3 c} \geq \frac{3 c^{2}}{2}|A|$. Suppose that the number of chunks of span at most $\frac{16}{c^{2}}$ is at most $\frac{23 c^{2}}{16}|A|$. Then $|A|>\frac{16}{c^{2}}\left(\frac{3 c^{2}}{2}-\frac{23 c^{2}}{16}\right)|A|=|A|$, a contradiction. Thus, we see that the number of chunks of span at most $\frac{16}{c^{2}}$ is at least $\frac{23 c^{2}}{16}|A|$. We call such short chunks good and since there are many such chunks, we consider only those which are good.

We now mark chunks that are at the desired distance apart within $A$. Start at the beginning of $A$ (in terms of the original labeling) and mark the first good chunk. We skip the next $\frac{64(t+2)}{c^{2}}$ vertices. We then mark the next good chunk which starts after the skipped vertices and repeat this process until we have crossed the entire length of $A$. Note that at most $\frac{64(t+2)}{c^{2}} / \frac{4}{3 c}+1=\frac{48(t+2)}{c}+1$ good chunks are contained in the skipped vertices. 
Certainly there are at least $\frac{23 c^{2}}{16}|A| /\left(\frac{48(t+2)}{c}+2\right)=\frac{23 c^{3}|A|}{16(48 t+96+2 c)}$ marked chunks.

Consider any segment of $\frac{\epsilon}{4 t^{2}} n$ consecutive vertices of $A$ and suppose that any such segments has at most $\frac{4}{c}$ marked chunks. Then the total number of marked chunks is at most $\frac{8}{c}\left(|A| / \frac{\epsilon}{4 t^{2}} n\right)=\frac{32 t^{2}|A|}{c \epsilon n}$. Therefore $\frac{32 t^{2}|A|}{c \epsilon n} \geq$ $\frac{23 c^{3}|A|}{16(48 t+96+2 c)}$, or $n \leq \frac{16 \cdot 32 t^{2}(48 t+96+2 c)}{23 c^{4} \epsilon}$, which contradicts the assumption $n$ is sufficiently large. So there must exist a segment containing at least $\frac{4}{c}$ marked chunks.

By Claim 7, there exists a swapping structure within these chunks. This is the desired swapping structure since (using the notation from Figure 2)

$$
\begin{aligned}
a_{2}-b_{2} & \geq \frac{64(t+2)}{c^{2}}-\frac{16}{c^{2}}=\frac{64 t+112}{c^{2}}, \\
a_{1}+a_{3}+b_{1}+b_{3} & \leq 2 \frac{16}{c^{2}}+2 \frac{4}{c}=\frac{32+8 c}{c^{2}}, \\
a_{1}+a_{2}+a_{3}-b_{2} & \leq \frac{\epsilon}{4 t^{2}} n+2 \cdot \frac{16}{c^{2}}<\frac{\epsilon}{2 t^{2}} n, \quad \text { and } \\
a_{2}-\left(b_{1}+b_{2}+b_{3}\right) & \leq \frac{\epsilon}{4 t^{2}} n<\frac{\epsilon}{2 t^{2}} n
\end{aligned}
$$

for $n$ sufficiently large.

As mentioned before, we obtain two new paths $P_{i}^{\prime}$ and $P_{j}^{\prime}$ within this structure. Let $\mathscr{P}^{\prime}=\left(\mathscr{P}-\left\{P_{i}, P_{j}\right\}\right) \cup\left\{P_{i}^{\prime}, P_{j}^{\prime}\right\}$. Since $\left|P_{j}\right|=\gamma_{j} n-f\left(P_{j}\right) \geq$ $\gamma_{j} n-f\left(P_{k+1}\right)>\gamma_{j} n+\frac{\epsilon}{t^{2}} n$, note that $\left|P_{j}^{\prime}\right|=\left|P_{j}\right|-\left(a_{1}+a_{2}+a_{3}\right)+b_{2}>$ $\gamma_{j} n+\frac{\epsilon}{t^{2}} n-\frac{\epsilon}{2 t^{2}} n \geq \epsilon n$. We know that $\left|P_{i}^{\prime}\right|=\left|P_{i}\right|-\left(b_{1}+b_{2}+b_{3}\right)+a_{2}>\left|P_{i}\right| \geq \epsilon n$. So $\left|P_{l}^{\prime}\right| \geq \epsilon n$ for any $P_{l}^{\prime} \in \mathscr{P}^{\prime}$. Now we calculate the difference $\mu(\mathscr{P})-\mu\left(\mathscr{P}^{\prime}\right)$.

We reorder paths in $\mathscr{P}^{\prime}$ and rename them $Q_{l}$ 's so that $f\left(Q_{l}\right) \geq f\left(Q_{l+1}\right)$ for all $1 \leq l \leq t-1$. Define a mapping $\phi$ from $\{1,2, \ldots, t\}$ to $\{1,2, \ldots, t\}$ so that $Q_{\phi(l)}=P_{l}$ for $l \neq i, j$ and $Q_{\phi(i)}=P_{i}^{\prime}$ and $Q_{\phi(j)}=P_{j}^{\prime}$. By the definition of $P_{i}^{\prime}$ and $P_{j}^{\prime}$,

$$
\begin{aligned}
f\left(P_{i}^{\prime}\right)-f\left(P_{j}^{\prime}\right)= & \gamma_{i} n-\left|P_{i}^{\prime}\right|-\gamma_{j} n+\left|P_{j}^{\prime}\right| \\
= & \gamma_{i} n-\gamma_{j} n-\left(\left|P_{i}\right|-\left(b_{1}+b_{2}+b_{3}\right)+a_{2}\right) \\
& +\left(\left|P_{j}\right|-\left(a_{1}+a_{2}+a_{3}\right)+b_{2}\right) \\
= & f\left(P_{i}\right)-f\left(P_{j}\right)-\left(a_{1}+a_{2}+a_{3}-b_{2}\right) \\
& -\left(a_{2}-\left(b_{1}+b_{2}+b_{3}\right)\right) \\
> & \left(\frac{\epsilon}{t^{2}}-2 \frac{\epsilon}{2 t^{2}}\right) n \\
= & 0 .
\end{aligned}
$$


This implies that $f\left(P_{i}^{\prime}\right)>f\left(P_{j}^{\prime}\right)$ and hence $\phi(i)<\phi(j)$. So,

$$
\phi(l)= \begin{cases}l & \text { for } 1 \leq l \leq i-1, \text { or } \phi(i)+1 \leq l \leq \phi(j)-1 \\ & \quad \text { or } j+1 \leq l \leq t \\ l-1 & \text { for } i+1 \leq l \leq \phi(i) \\ l+1 & \text { for } \phi(j) \leq l \leq j-1\end{cases}
$$
Since

Denote $g\left(Q_{\phi(l)}\right):=\gamma_{l} n-\left|Q_{\phi(l)}\right|$. Then $\mu(\mathscr{Q})=\sum_{l=1}^{t}(t-\phi(l)+1) g\left(Q_{\phi(l)}\right)$.

$$
\mu(\mathscr{P})-\mu(\mathscr{Q})=\sum_{l=1}^{t}\left((t-l+1) f\left(P_{l}\right)-(t-\phi(l)+1) g\left(Q_{\phi(l)}\right)\right),
$$

it suffices to calculate each member of the right side. By the definition of $\phi$, for $1 \leq l \leq i-1$, or $\phi(i)+1 \leq l \leq \phi(j)-1$ or $j+1 \leq l \leq t$,

$$
\begin{aligned}
& (t-l+1) f\left(P_{l}\right)-(t-\phi(l)+1) g\left(Q_{\phi(l)}\right) \\
& \quad=(t-l+1) f\left(P_{l}\right)-(t-l+1) f\left(P_{l}\right) \\
& \quad=0 .
\end{aligned}
$$

For $i+1 \leq l \leq \phi(i)$

$$
\begin{aligned}
& (t-l+1) f\left(P_{l}\right)-(t-\phi(l)+1) g\left(Q_{\phi(l)}\right) \\
& \quad=(t-l+1) f\left(P_{l}\right)-(t-l+2) f\left(P_{l}\right) \\
& \quad=-f\left(P_{l}\right)
\end{aligned}
$$

and for $\phi(j) \leq l \leq j-1$,

$$
\begin{aligned}
& (t-l+1) f\left(P_{l}\right)-(t-\phi(l)+1) g\left(Q_{\phi(l)}\right) \\
& \quad=(t-l+1) f\left(P_{l}\right)-(t-l) f\left(P_{l}\right) \\
& \quad=f\left(P_{l}\right) .
\end{aligned}
$$

For $l=i$,

$$
\begin{aligned}
(t- & i+1) f\left(P_{i}\right)-(t-\phi(i)+1) g\left(Q_{\phi(i)}\right) \\
= & (t-i+1)\left(\gamma_{i} n-\left|P_{i}\right|\right)-(t-\phi(i)+1)\left(\gamma_{i} n-\left|Q_{\phi(i)}\right|\right) \\
= & (t-i+1)\left(\gamma_{i} n-\left|P_{i}\right|\right) \\
& \quad-(t-\phi(i)+1)\left(\gamma_{i} n-\left|P_{i}\right|+\left(b_{1}+b_{2}+b_{3}\right)-a_{2}\right)
\end{aligned}
$$




$$
\begin{aligned}
& =(\phi(i)-i)\left(\gamma_{i} n-\left|P_{i}\right|\right)-(t-\phi(i)+1)\left(b_{1}+b_{2}+b_{3}-a_{2}\right) \\
& =(\phi(i)-i) f\left(P_{i}\right)-(t-\phi(i)+1)\left(b_{1}+b_{2}+b_{3}-a_{2}\right),
\end{aligned}
$$

and for $l=j$,

$$
\begin{aligned}
(t- & j+1) f\left(P_{j}\right)-(t-\phi(j)+1) g\left(Q_{\phi(j)}\right) \\
= & (t-j+1)\left(\gamma_{j} n-\left|P_{j}\right|\right)-(t-\phi(j)+1)\left(\gamma_{j} n-\left|Q_{\phi(j)}\right|\right) \\
= & (t-j+1)\left(\gamma_{j} n-\left|P_{j}\right|\right) \\
& -(t-\phi(j)+1)\left(\gamma_{j} n-\left|P_{j}\right|+\left(a_{1}+a_{2}+a_{3}\right)-b_{2}\right) \\
= & -(j-\phi(j))\left(\gamma_{j} n-\left|P_{j}\right|\right)-(t-\phi(j)+1)\left(a_{1}+a_{2}+a_{3}-b_{2}\right) \\
= & -(j-\phi(j)) f\left(P_{j}\right)-(t-\phi(j)+1)\left(a_{1}+a_{2}+a_{3}-b_{2}\right),
\end{aligned}
$$

Therefore, Equations (1)-(5) imply

$$
\begin{aligned}
\mu(\mathscr{P})-\mu(\mathscr{Q})= & -(t-\phi(i)+1)\left(b_{1}+b_{2}+b_{3}-a_{2}\right) \\
& -(t-\phi(j)+1)\left(a_{1}+a_{2}+a_{3}-b_{2}\right)-\sum_{l=i+1}^{\phi(i)} f\left(P_{l}\right) \\
& +\sum_{l=\phi(j)}^{j-1} f\left(P_{l}\right)+(\phi(i)-i) f\left(P_{i}\right)-(j-\phi(j)) f\left(P_{j}\right) .
\end{aligned}
$$

Since $f\left(P_{l}\right) \leq f\left(P_{i}\right)$ for any $i+1 \leq l \leq \phi(i)$, we have $\sum_{l=i+1}^{\phi(i)} f\left(P_{l}\right) \leq$ $(\phi(i)-i) f\left(P_{i}\right)$, and since $f\left(P_{l}\right) \geq f\left(P_{j}\right)$ for any $\phi(j) \leq l \leq j-1$,

$$
\sum_{l=\phi(j)}^{j-1} f\left(P_{l}\right) \geq(j-\phi(j)) f\left(P_{j}\right) .
$$

So,

$$
\begin{aligned}
\mu(\mathscr{P})-\mu(\mathscr{Q}) \geq & -(t-\phi(i)+1)\left(b_{1}+b_{2}+b_{3}-a_{2}\right) \\
& -(t-\phi(j)+1)\left(a_{1}+a_{2}+a_{3}-b_{2}\right) \\
= & (\phi(j)-\phi(i))\left(a_{2}-b_{2}\right) \\
& -(t-\phi(i)+1)\left(a_{1}+a_{3}\right)-(t-\phi(j)+1)\left(b_{1}+b_{3}\right) \\
\geq & \left(a_{2}-b_{2}\right)-t\left(a_{1}+a_{3}+b_{1}+b_{3}\right) \\
\geq & \frac{64 t+112}{c^{2}}-t\left(a_{1}+a_{3}+b_{1}+b_{3}\right) .
\end{aligned}
$$


Now we apply Lemma 1 and we get a spanning path collection $\mathscr{Q}^{\prime}$. Since $\left|Q_{l}\right| \geq \epsilon n$ for all $1 \leq l \leq t$ and $|V(G)|-\sum_{l=1}^{t}\left|P_{l}\right| \leq a_{1}+a_{3}+b_{1}+b_{3} \leq$ $\frac{32+8 c}{c^{2}} \leq \frac{n}{8}$, Lemma 1 can work for $\mathscr{Q}$. We now show that $\mu\left(\mathscr{Q}^{\prime}\right)<\mu(\mathscr{P})$. The upper bound of increase from $\mathscr{Q}$ to $\mathscr{Q}^{\prime}$ is at most $t$ times the number of vertices which may be added to paths, because $t$ is the maximum weight over the sum of $\mu$. In particular, in the application of Lemma 1, we add at most $a_{1}+a_{3}+b_{1}+b_{3}$ since we do not have control over where these vertices fall and Lemma 1 adds at most an additional $\frac{20}{\epsilon^{2}}$ vertices. Hence,

$$
\mu\left(\mathscr{Q}^{\prime}\right)-\mu(\mathscr{Q}) \leq t \cdot\left(a_{1}+a_{3}+b_{1}+b_{3}\right)+t \cdot \frac{20}{\epsilon^{2}}
$$

Thus, using Inequalities (6) and (7), we get

$$
\begin{aligned}
\mu(\mathscr{P})-\mu\left(\mathscr{Q}^{\prime}\right) & \geq \frac{64 t+112}{c^{2}}-2 t\left(a_{1}+a_{3}+b_{1}+b_{3}\right)-t \cdot \frac{20}{\epsilon^{2}} \\
& \geq \frac{64 t+112}{c^{2}}-\frac{64 t+16 c t}{c^{2}}-\frac{20 t}{\epsilon^{2}} \\
& =\frac{112}{c^{2}}-\frac{16 t}{c}-\frac{20 t}{\epsilon^{2}} \\
& \geq \frac{112 \cdot 24^{2} \cdot 48^{2} t^{4}}{\epsilon^{4}}-\frac{16 \cdot 24 \cdot 48 t^{3}}{\epsilon^{2}}-\frac{20 t}{\epsilon^{2}} \\
& >0,
\end{aligned}
$$

because $c=\frac{\epsilon^{2}}{24 \cdot 48 t^{2}}$ and $\epsilon \leq \frac{1}{6 t(2 t+1)}$. Thus, $\mu\left(\mathscr{Q}^{\prime}\right)<\mu(\mathscr{P})$. This completes the proof of Lemma 2.

Our next lemma constructs the desired path system directly if the conditions of Lemma 2 are not satisfied. The proof is similar to the proof of the corresponding Rebuilding Lemma in [8].

Lemma 3 (Rebuilding). Given a set of fractions $\gamma_{1}, \ldots, \gamma_{t}$, let $c_{1}$ and $c_{2}$ be positive real numbers with $c_{1} \leq \frac{\epsilon}{24}$ and $c_{2}<\min \left\{\frac{c_{1}}{3}, \gamma_{i} / 16\right\}$ for all $i$. Suppose that $n$ is sufficiently large (compared to $c_{1}$ and $c_{2}$ ). If $\kappa(G) \geq 2 t+1$, $\sigma_{2}(G) \geq n+2 t-2$ and $V(G)$ can be partitioned into sets $A$ and $B$ with $|A|,|B| \geq\left(4 c_{1}+c_{2}\right) n$ and $e(A, B)<c_{1} c_{2} n^{2}$ then $G$ contains a spanning desired path collection for $S$.

Proof. Let $D_{A}$ be the set of vertices in $A$ with at least $c_{1} n$ edges into $B$ and let $D_{B}$ be the set of vertices in $B$ with at least $c_{1} n$ edges into $A$. We know $e(A, B)<c_{1} c_{2} n^{2}$ so $\left|D_{A}\right|,\left|D_{B}\right|<c_{2} n$. Let $A^{\prime}:=A \backslash D_{A}, B^{\prime}:=B \backslash D_{B}$ and let $u \in A^{\prime}$ and $v \in B^{\prime} \backslash N(u)$. Since $d_{B}(u)<c_{1} n$, for every choice of $u$, 
there exists such a $v$. The edge $u v \notin E(G)$ so $d(u)+d(v) \geq n+2 t-2$ but $d_{B}(u), d_{A}(v)<c_{1} n$ so $d_{A}(u)+d_{B}(v)>n-2 c_{1} n+2 t-2$. In particular, this implies that for all vertices $u \in A^{\prime}$, we have $d_{A}(u)>|A|-2 c_{1} n+2 t-2$. By symmetry $d_{B}(v)>|B|-2 c_{1} n+2 t-2$ for all $v \in B^{\prime}$.

Claim 8. For any $\tilde{A} \subseteq A^{\prime}$ with $|\tilde{A}| \geq 4 c_{1} n, G[\tilde{A}]$ is panconnected, and for any $\tilde{B} \subseteq B^{\prime}$ with $|\tilde{B}| \geq 4 c_{1} n, G[\tilde{B}]$ is panconnected. In particular, $G\left[A^{\prime}\right]$ and $G\left[\overline{B^{\prime}}\right]$ are panconnected. Also, this means that if $|\tilde{A}| \geq \frac{\epsilon n}{6}$, then $G[\tilde{A}]$ is panconnected and similarly for $\tilde{B}$.

Proof of Claim 8. We now recall that $d_{A}(v)>|A|-2 c_{1} n+2 t-2$ for any $v \in A^{\prime}$. Therefore

$$
\begin{aligned}
\delta(G[\tilde{A}]) & >|A|-2 c_{1} n+2 t-2-|A \backslash \tilde{A}| \\
& =|\tilde{A}|-2 c_{1} n+2 t-2 \\
& \geq \frac{|\tilde{A}|}{2}+2 t-2 .
\end{aligned}
$$

By Theorem 7 we know $G[\tilde{A}]$ is panconnected. In particular, $\left|A^{\prime}\right|=$ $|A|-\left|D_{A}\right| \geq\left(4 c_{1}+c_{2}\right) n-c_{2} n=4 c_{1} n$, which implies that $G\left[A^{\prime}\right]$ is panconnected. By the symmetry, both $G[\tilde{B}]$ and $G\left[B^{\prime}\right]$ are also panconnected.

$\square_{\text {Claim } 8}$

Now, by Menger's Theorem [12], there is a matching $\mathscr{M}$ between $A^{\prime} \cup D_{B}$ and $B^{\prime} \cup D_{A}$ in $G$ with $|\mathscr{M}|=2 t+1$. Choose such $\mathscr{M}$ so that $|V(\mathscr{M}) \cap S|$ is as small as possible. Notice $\mathscr{M}$ contains exactly $2 t+1$ vertices of $A^{\prime} \cup D_{B}$ and $2 t+1$ vertices of $B^{\prime} \cup D_{A}$. Let $\mathscr{M} \cap\left(A^{\prime} \cup D_{B}\right):=\left\{a_{1}, a_{2}, \ldots, a_{2 t+1}\right\}$ and $\mathscr{M} \cap\left(B^{\prime} \cup D_{A}\right):=\left\{b_{1}, b_{2}, \ldots, b_{2 t+1}\right\}$ so that $a_{i} b_{i} \in \mathscr{M}$. The choice of $\mathscr{M}$ implies the following claim.

Claim 9. Every edge in $\mathscr{M}$ has at most one vertex in $S$ or $G$ contains a spanning path collection for $S$.

Proof of Claim 9. Let $u \in A^{\prime} \cup D_{B} \backslash(V(\mathscr{M}) \cup S)$ and let $v \in B^{\prime} \cup D_{A} \backslash$ $(V(\mathscr{M}) \cup S)$. Since $\left|A^{\prime}\right| \geq 4 c_{1} n>4 t+1$, there exists such a vertex $u \in A^{\prime}$, and by symmetry there exists such a vertex $v \in B^{\prime}$. If $u v \in E(G)$, then no edge in $\mathscr{M}$ contains a vertex in $S$. (Otherwise we can switch out such an edge for the edge $u v$.) Thus, we may assume that $u v \notin E(G)$.

If there exists $w \in(N(u) \cap N(v)) \backslash V(\mathscr{M})$, then no edge in $\mathscr{M}$ contains two vertices in $S$. (Again otherwise we can change such an edge with an edge $u w$ or $v w$.) So we may assume that

$$
N(u) \cap N(v) \subseteq\left\{a_{1}, a_{2}, \ldots, a_{2 t+1}, b_{1}, \ldots, b_{2 t+1}\right\} .
$$


If $a_{j} \in N(u) \cap N(v)$, then $b_{j} \notin S$; otherwise we can change an edge $a_{j} b_{j}$ with an edge $a_{j} v$. Suppose that $a_{j}, b_{j} \in N(u) \cap N(v)$ for some $j$. By the above fact, $a_{j}, b_{j} \notin S$. Moreover, if there exists an edge $a_{k} b_{k} \in \mathscr{M}$ such that $a_{k} \in S$ or $b_{k} \in S$, then we can change two edges $a_{j} b_{j}$ and $a_{k} b_{k}$ with two edges $a_{j} v$ and $u b_{j}$, which contradicts the choice of $\mathscr{M}$. Therefore, no edge in $\mathscr{M}$ contains a vertex in $S$, so we may assume that $a_{j} \notin N(u) \cap N(v)$ or $b_{j} \notin N(u) \cap N(v)$.

If $|N(u) \cap N(v)| \leq 2 t-1$, then $d(u)+d(v)=|N(u) \cup N(v)|+\mid N(u) \cap$ $N(v) \mid \leq n+2 t-3$, a contradiction. Thus, $\left|N_{G}(u) \cap N_{G}(v)\right| \geq 2 t$. Hence by changing the index if necessary, we may assume that $a_{j} \in N(u) \cap N(v)$ or $b_{j} \in N(u) \cap N(v)$ for all $2 \leq j \leq 2 t+1$. This implies that $a_{j} \notin S$ or $b_{j} \notin S$ for all $2 \leq j \leq 2 t+1$. If $a_{1} \notin S$ or $b_{1} \notin S$, then we obtain the conclusion of the claim. Hence we may again assume that $a_{1} \in S$ and $b_{1} \in S$. In this case, all the above inequalities hold, so $N(u) \cup N(v)=V(G)-\{u, v\}$. Since $N(u) \subset A^{\prime} \cup D_{B} \cup\left\{b_{1}, \ldots, b_{2 t+1}\right\}$, we obtain $B^{\prime} \cup D_{A} \subset N(v)$. By symmetry, $A^{\prime} \cup D_{B} \subset N(u)$. Because this condition holds for any $u \in$ $A^{\prime} \cup D_{B} \backslash(V(\mathscr{M}) \cup S)$, any two vertices of $A^{\prime} \cup D_{B}$ are connected by an edge except for two vertices in $V(\mathscr{M}) \cup S$. By symmetry, any two vertices of $B^{\prime} \cup D_{A}$ are connected by an edge except for two vertices in $V(\mathscr{M}) \cup S$. Construction of the desired paths is trivial in this situation. This completes the proof of the claim.

Claim 9

Let $v \in D_{B}$. Since $\left|D_{A}\right| \leq c_{2} n$ and $d_{A}(v) \geq c_{1} n$, we have $d_{A^{\prime}}(v) \geq$ $d_{A}(v)-\left|D_{A}\right|>\left(c_{1}-c_{2}\right) n$. Then the conditions " $c_{1}>c_{2}$ " and " $n$ is sufficiently large" implies the existence of a matching $\mathscr{M}_{A}$ between $V(\mathscr{M}) \cap D_{B}$ and $A^{\prime} \backslash$ $(V(\mathscr{M}) \cup S)$. By the symmetry, we have a matching $\mathscr{M}_{B}$ between $V(\mathscr{M}) \cap D_{A}$ and $B^{\prime} \backslash(V(\mathscr{M}) \cup S)$.

For any $a_{j} \in V(\mathscr{M}) \cap D_{B}$, let $a_{j} a_{j}^{\prime}$ be an edge in the matching $\mathscr{M}_{A}$ and for any $b_{j} \in V(\mathscr{M}) \cap D_{A}$, let $b_{j} b_{j}^{\prime}$ be an edge in the matching $\mathscr{M}_{B}$. For convenience, let $a_{i}^{\prime}=a_{i}$ if $a_{i} \in V(\mathscr{M}) \cap A^{\prime}$ and $b_{i}^{\prime}=b_{i}$ if $b_{i} \in V(\mathscr{M}) \cap B^{\prime}$.

Let $Q_{j}:=a_{j}^{\prime}\left(a_{j}\right)\left(b_{j}\right) b_{j}^{\prime}$. Notice that $Q_{j}$ is a path of length one, two or three, and has at most one vertex in $S$. Let $\mathscr{Q}:=\left\{Q_{j}: 1 \leq j \leq 2 t+1\right\}$. The paths in $\mathscr{Q}$ will be used for "transportation" from $A^{\prime}$ to $B^{\prime}$ when we construct the desired paths.

If $x_{i} \in D_{A}$ (resp. $y_{i} \in D_{A}$ ), then we take a vertex $x_{i}^{\prime}$ (resp. $y_{i}^{\prime}$ ) in $B^{\prime} \backslash(S \cup V(\mathscr{Q}))$. Since $d_{B^{\prime}}(v) \geq\left(c_{1}-c_{2}\right) n$ for any $v \in D_{A}$, we can take such vertices $x_{i}^{\prime}$ and $y_{i}^{\prime}$ so that all of $x_{i}^{\prime}$ 's and $y_{i}^{\prime}$ 's are distinct. We similarly define $x_{i}^{\prime}$ and $y_{i}^{\prime}$ in $A^{\prime}$ for $x_{i}, y_{i} \in D_{B}$. For convenience, let $x_{i}^{\prime}=x_{i}$ and let $y_{i}^{\prime}=y_{i}$ for $x_{i}, y_{i} \in A^{\prime} \cup B^{\prime}$. 


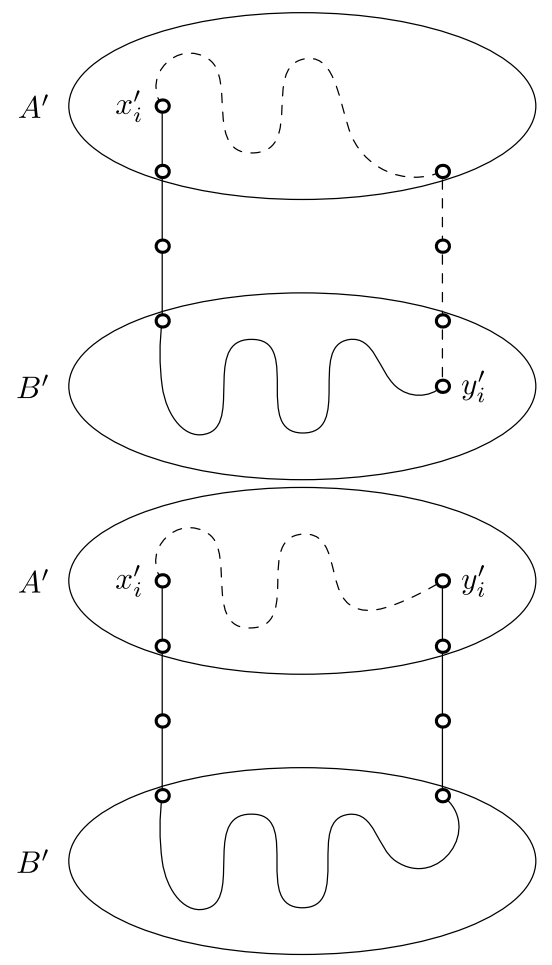

Figure 3: Routing paths.

We now provide a process for constructing the desired path collection. Without loss of generality, we may assume that $\gamma_{1} \leq \gamma_{2} \leq \cdots \leq \gamma_{t}$. The paths are constructed as follows. Before constructing formally, we show the outline of the proof.

For each $1 \leq i \leq t-1$ start at the vertex $x_{i}^{\prime}$ (suppose $x_{i}^{\prime}$ is in $A^{\prime}$ ). Suppose that $y_{i}^{\prime} \in B^{\prime}$. Then we can choose a desired path from two choices depending on the length of $\gamma_{i}$. One of them is starting from $x_{i}^{\prime}$, "traversing" $A^{\prime}$ until attaining the appropriate length, "jumping" to $B^{\prime}$ and ending at $y_{i}^{\prime}$; the other "traverses" $B^{\prime}$ after "jumping" to $B^{\prime}$. If $\gamma_{i}$ is too small for our path to "fit" into $A^{\prime}$, then we choose the first option; otherwise we choose the second. If $y_{i}^{\prime} \in A^{\prime}$, then there are also two choices, one of them "traverses" $A^{\prime}$ without "jumping"; another "jumps" to $B^{\prime}$, "traverses" $B^{\prime}$ and "jumps" back to $A^{\prime}$. In each case, we create such a path using no more than two paths in $\mathscr{Q}$. After constructing $t-1$ paths, we finally construct a path $P_{t}$. Since at least three paths in $\mathscr{Q}$ remain, we can "traverse" both $A^{\prime}$ and $B^{\prime}$ until attaining the appropriate length. Figure 3 depicts the two cases listed above and their two subcases for constructing these paths. 
Formally, we will show the existence of a spanning desired path collection. We first connect the vertices of $D_{A} \backslash \mathscr{Q}$ into a path. Since each vertex of $D_{A}$ has at least $c_{1} n$ edges to $B$ and $\left|D_{A}\right|<c_{2} n<c_{1} n / 3$, there must exist a system of two distinct neighbors in $B$ of each vertex of $D_{A}$. By Claim 8 , we may easily connect these vertices by short paths in $B^{\prime}$ so that all of $D_{A} \backslash \mathscr{Q}$ is on a single path of length at most $4 c_{2} n<\gamma_{i} n / 4$ which begins and ends in $B^{\prime}$. The same can be said for $D_{B} \backslash \mathscr{Q}$ so, when constructing the desired paths, we simply use the path through $D_{A} \backslash \mathscr{Q}$ the first time we use vertices of $B^{\prime}$ and use the path through $D_{B} \backslash \mathscr{Q}$ the first time we use vertices of $A^{\prime}$.

Suppose we have constructed paths $P_{1}, \ldots, P_{i-1}$ for $1 \leq i \leq t-1$ such that each of them contains vertices in at most two paths in $\mathscr{Q}$ and $\left(\gamma_{j}-\right.$ $\left.\frac{\epsilon}{2 t}\right) n \leq\left|P_{j}\right| \leq\left(\gamma_{j}-\frac{\epsilon}{2 t}\right) n+4$ for all $j \leq i-1$. We would like to construct $P_{i}$. Let $R_{i}=V\left(P_{1}\right) \cup \cdots \cup V\left(P_{i-1}\right)$ and let $\tilde{A}=\left[A^{\prime} \backslash\left(S \cup R_{i} \cup V(\mathscr{Q})\right)\right]$ and let $\tilde{B}=\left[B^{\prime} \backslash\left(S \cup R_{i} \cup V(\mathscr{Q})\right)\right]$. By symmetry, we may assume that $x_{i}^{\prime} \in A^{\prime}$. If $\left(\gamma_{i}-\frac{\epsilon}{2 t}\right) n \leq|\tilde{A}|-\frac{\epsilon}{6} n$, use Claim 8 to complete the path $P_{i}$ of order $\left(\gamma_{i}-\frac{\epsilon}{2 t}\right) n$ in $A^{\prime}$ from $x_{i}^{\prime}$ to $a_{j}^{\prime}$, where $a_{j}^{\prime}$ is chosen so that $y_{i}^{\prime} \in V\left(Q_{j}\right)$ if $y_{i}^{\prime} \in V(\mathscr{Q})$; otherwise $Q_{j}$ is not used in the previous paths $P_{1}, \ldots, P_{i-1}$ and does not contain a vertex in $S$. Since each path in $\mathscr{Q}$ contains at most one vertex in $S$ and at most $2(i-1)$ paths in $\mathscr{M}$ are used for the previous paths $P_{1}, \ldots, P_{i-1}$, we can find such $Q_{j}$. If $y_{i}^{\prime} \in A^{\prime}$, construct a short path (of length at most 2) from $a_{j}^{\prime}$ to $y_{i}^{\prime}$ in $A^{\prime}$ and finish with the path to $y_{i}$. Otherwise take the path $Q_{j}$ from $a_{j}^{\prime}$ to $b_{j}^{\prime}$ (or $y_{i}^{\prime}$ if $y_{i}^{\prime} \in V\left(Q_{j}\right) \backslash\left\{b_{j}^{\prime}\right\}$ ) and take a path of length at most 2 in $B^{\prime} \cup D_{A}$ from $b_{j}^{\prime}$ to $y_{i}^{\prime}$. Notice $\left|\tilde{A} \backslash P_{i}\right| \geq$ $|\tilde{A}|-\left(\gamma_{i}-\frac{\epsilon}{2 t}\right) n>\frac{\epsilon}{6} n$ and since $\frac{\epsilon}{6} \geq 4 c_{1}$, we see that $G\left[\tilde{A} \backslash P_{i}\right]$ is still panconnected.

Suppose that $\left(\gamma_{i}-\frac{\epsilon}{2 t}\right) n>|\tilde{A}|-\frac{\epsilon}{6} n$. Then $\left|R_{i} \cap A^{\prime}\right| \geq\left|A^{\prime}\right|-|\tilde{A}|-|S|-$ $|V(\mathscr{Q})|>\left|A^{\prime}\right|-\left(\gamma_{i}-\frac{\epsilon}{2 t}\right) n-\frac{\epsilon}{6} n-6 t$. Hence

$$
\begin{aligned}
\left|R_{i} \cap B^{\prime}\right| & \leq \sum_{j=1}^{i-1}\left|P_{j}\right|-\left|R_{i} \cap A^{\prime}\right| \\
& <\sum_{j=1}^{i-1}\left(\left(\gamma_{j}-\frac{\epsilon}{2 t}\right) n+4\right)-\left|A^{\prime}\right|+\left(\gamma_{i}-\frac{\epsilon}{2 t}\right) n+\frac{\epsilon}{6} n+6 t \\
& \leq\left(\sum_{j=1}^{i} \gamma_{j}\right) n-\frac{i \epsilon}{2 t} n+4(i-1)+\frac{\epsilon}{6} n-\left(n-\left|B^{\prime}\right|-\frac{\epsilon}{36} n\right)+6 t \\
& =\left|B^{\prime}\right|-\left(\sum_{j=i+1}^{t} \gamma_{j}\right) n-\frac{i \epsilon}{2 t} n+4(i-1)+\frac{7 \epsilon}{36} n+6 t
\end{aligned}
$$


which implies:

$$
\begin{aligned}
|\tilde{B}|-\frac{\epsilon}{6} n & =\left|B^{\prime}\right|-\left|R_{i} \cap B^{\prime}\right|-\frac{\epsilon}{6} n \\
& >\left(\sum_{j=i+1}^{t} \gamma_{j}\right) n+\frac{i \epsilon}{2 t} n-4(i-1)-\frac{7 \epsilon}{36} n-6 t-\frac{\epsilon}{6} n \\
& >\left(\sum_{j=i+1}^{t} \gamma_{j}\right) n+\frac{i \epsilon}{2 t} n-4(i-1)-\frac{3 \epsilon}{8} n \\
& \geq \gamma_{t} n+\frac{t-i-1}{2 t} \epsilon n+\frac{i \epsilon}{2 t} n-4(i-1)-\frac{3 \epsilon}{8} n \\
& =\gamma_{t} n+\frac{t-1}{2 t} \epsilon n-4(i-1)-\frac{3 \epsilon}{8} n \\
& \geq \gamma_{t} n-\frac{1}{2 t} \epsilon n \\
& =\left(\gamma_{i}-\frac{\epsilon}{2 t}\right) n
\end{aligned}
$$

We use the fact that $\tilde{A}$ is panconnected to create a path from $x_{i}^{\prime}$ to $a_{j}^{\prime}$ of length 2 for some $j$ such that $a_{j}^{\prime}$ is not contained in $R_{i} \cup S$ except for $x_{i}^{\prime}$. We construct this short path from $x_{i}^{\prime}$ to $a_{j}^{\prime}$ to preserve the panconnectivity of $\tilde{A}$ and connect $x_{i}^{\prime}$ to $Q_{j}$. Since $\left(\gamma_{i}-\frac{\epsilon}{2 t}\right) n<|\tilde{B}|-\frac{\epsilon}{6} n$, we can use the panconnectivity of $\tilde{B}$ to complete the path $P_{i}$ of length $\left(\gamma_{i}-\frac{\epsilon}{2 t}\right) n$ to $y_{i}^{\prime}$ if $y_{i}^{\prime} \in B^{\prime}$. If $y_{i}^{\prime} \in A^{\prime}$, then take $a_{k}^{\prime}$ so that $Q_{k}$ is not used in $R_{i} \cup Q_{j}$. In particular, we choose $Q_{k}$ so that $a_{k}^{\prime}=y_{i}^{\prime}$ if possible. After that, $G\left[\tilde{B} \backslash P_{i}\right]$ is still panconnected since $\left|\tilde{B} \backslash P_{i}\right| \geq \frac{\epsilon n}{6}$.

Suppose we have constructed $t-1$ paths as above. Let $R_{t}=V\left(P_{1}\right) \cup \cdots \cup$ $V\left(P_{t-1}\right)$. Without loss of generality, suppose $x_{t}^{\prime} \in A^{\prime}$ and let $Q_{1}, Q_{2}, Q_{3}$ be remaining paths of $\mathscr{Q}$. Such paths exist because we use at most two paths of $\mathscr{Q}$ to construct each path $P_{i}$ and $|\mathscr{Q}|=2 t+1$. Let $\tilde{A}=\left[A^{\prime} \backslash\left(S \cup R_{t} \cup V(\mathscr{Q})\right)\right]$ and let $\tilde{B}=\left[B^{\prime} \backslash\left(S \cup R_{t} \cup V(\mathscr{Q})\right)\right]$ and note that $|\tilde{A}|,|\tilde{B}| \geq \frac{\epsilon n}{6}$ so, by Claim 8 , both sets are still panconnected. By symmetry, we may assume that $x_{t}^{\prime} \neq a_{1}^{\prime}, a_{2}^{\prime}$. If $y_{t}^{\prime}$ are in $A^{\prime}$, (suppose $y_{t}^{\prime} \neq a_{2}^{\prime}$ ) use the panconnectivity of $G[\tilde{A}]$ to connect $x_{t}^{\prime}$ to $a_{1}^{\prime}$ using at most one intermediate vertex and connect $y_{t}^{\prime}$ to $a_{2}^{\prime}$ using all of $\tilde{A}$. We then take the paths, through $Q$, to $\tilde{B}$ and use the panconnectivity of $\tilde{B}$ to pick up all of $B^{\prime}$ between $b_{1}^{\prime}$ and $b_{2}^{\prime}$. This produces a path of order $\left|P_{t}\right| \leq n-\sum_{j=1}^{t-1}\left|P_{i}\right|-|D| \leq \gamma_{t} n+\frac{2(t-1) \epsilon}{2 t} n+\frac{\epsilon}{36}$.

Otherwise suppose $y_{t}^{\prime} \in B^{\prime}$. By symmetry, $a_{1}^{\prime} \neq x_{t}^{\prime}$ and $b_{1}^{\prime} \neq y_{t}^{\prime}$. Again use the panconnectivity of $G\left[A \backslash R_{t}\right]$ and $G\left[B \backslash R_{t}\right]$ to connect $x_{t}^{\prime}$ to $a_{1}^{\prime}$ using all of $A^{\prime}$, through $Q_{1}$ and connect $b_{1}^{\prime}$ to $y_{t}^{\prime}$ using all of $B^{\prime}$. 
For any $P_{j} \in \mathscr{P}$, we have $\left|P_{j}\right|<\gamma_{j} n+\frac{2(t-1) \epsilon}{2 t} n+\frac{\epsilon}{24} \leq\left(\gamma_{j}+\epsilon\right) n$ for $n$ sufficiently large. On the other hand, $\left|P_{j}\right| \geq\left(\gamma_{j}-\epsilon\right) n$, which completes the proof of Lemma 3.

Our final lemma gives necessary starting structure for the proofs of our main results.

Lemma 4 (Setup). Suppose that $n$ is sufficiently large. If $\kappa(G) \geq 2 t+1$ and $\sigma_{2}(G) \geq n+2 t-2$, there exists a spanning path collection for $S$.

Proof. First we will show the existence of a path collection (possibly not spanning). After finding a path collection, we obtain the desired path collection by applying Lemma 1.

In order to show this, we divide the proof into two parts depending on the connectivity. Suppose first that $\kappa(G)<2 t \epsilon n$, and let $K$ be a minimum cutset of order less than $2 t \epsilon n$. Since $\sigma_{2}(G) \geq n+2 t-2$ and $\epsilon>0$ is small, $G \backslash K$ has exactly two components, so we know there exists a component $A$ of $G \backslash K$ with $|A| \geq \frac{(1-2 t \epsilon) n}{2}$. Let $B=V(G)-(A \cup K)$.

If $|B \cup K| \geq 9 n \sqrt{t \epsilon}$, then we know $e(A, B \cup K) \geq 2 t \epsilon n^{2}$, because otherwise we can apply Lemma 3 by letting $c_{1}:=2 \sqrt{t \epsilon}$ and $c_{2}:=\frac{\sqrt{t \epsilon}}{2}$. Since all these edges must be incident to vertices in $K$, there exists a vertex $v \in K$ such that,

$$
d_{A}(v)>\frac{2 t \epsilon n^{2}}{2 t \epsilon n}=n .
$$

So we may assume that $|B \cup K|<9 n \sqrt{t \epsilon}$. We know $d_{G}(v) \leq|B|+|K|-1$ for any $v \in B$, so $B$ induces a clique, because otherwise, $d_{G}\left(v_{1}\right)+d_{G}\left(v_{2}\right) \leq$ $2|B|+2|K|-2<18 n \sqrt{t \epsilon} \leq n$, a contradiction, where $v_{1}, v_{2} \in B$ such that $v_{1} v_{2} \notin E(G)$. Consider a vertex $v \in B$. Since $u \in A$ has $d_{G}(u) \geq$ $n+2 t-2-|B|-|K|=|A|+2 t-2, d_{A}(u) \geq|A|+2 t-2-|K|>|A|-2 t \epsilon n+2 t-2$. This implies that for any $\tilde{A} \subseteq A$ with $|\tilde{A}| \geq 4 t \epsilon n, \tilde{A}$ is panconnected, because $d_{\tilde{A}}(u) \geq|A|-2 t \epsilon n+2 t-|A \backslash \tilde{A}|=|\tilde{A}|-2 t \epsilon n+2 t-2 \geq \frac{|\tilde{A}|+2}{2}$.

Since $G$ is $2 t$-connected and $|(B \cup K) \cap S|=|S|-|A \cap S|=2 t-|A \cap S|$, there exists a collection of paths $\mathscr{Q}$ from $(B \cup K) \cap S$ to $A-(A \cap S)$ in $G-A \cap S$. For $x_{i} \in(B \cup K) \cap S\left(y_{i} \in(B \cup K) \cap S\right)$, let $Q_{x_{i}}\left(Q_{y_{i}}\right)$ be a path in $\mathscr{Q}$ starting from $x_{i}\left(y_{i}\right.$, respectively). Let $x_{i}^{\prime}\left(y_{i}^{\prime}\right)$ be another end vertex of $Q_{x_{i}}\left(Q_{y_{i}}\right)$.

For convenience, let $x_{i}^{\prime}:=x_{i}$ and $y_{i}^{\prime}:=y_{i}$ for any $x_{i}, y_{i} \in A \cap S$. We will show that there exist $t$ disjoint paths $P_{1}^{\prime}, P_{2}^{\prime}, \ldots, P_{t}^{\prime}$ such that $P_{i}^{\prime}$ connects $x_{i}^{\prime}$ and $y_{i}^{\prime}$ with $\left|P_{i}^{\prime}\right|=\epsilon n$. Suppose first that we have already found $i$ disjoint paths $P_{1}^{\prime}, P_{2}^{\prime}, \ldots, P_{i}^{\prime}$ for $i<t$. Let $\tilde{A}=A-c u p_{j=1}^{i} V\left(P_{j}^{\prime}\right)$. Note that 
$|\tilde{A}|=|A|-\sum_{j=1}^{i}\left|P_{j}^{\prime}\right|>(1-9 \sqrt{t \epsilon}) n-i \epsilon n \geq 4 t \epsilon n$, because $\epsilon<\frac{1}{18^{2} t}$ (which implies $1>4 t \epsilon+i \epsilon+9 \sqrt{t \epsilon})$. Hence $\tilde{A}$ is panconnected. This implies that we can find a path $P_{i+1}^{\prime}$ in $\tilde{A}$ connecting $x_{i+1}^{\prime}$ and $y_{i+1}^{\prime}$ with $\left|P_{i+1}\right|=\epsilon n$. By joining $Q_{x_{i}}, P_{i}^{\prime}$ and $Q_{y_{i}}$, we obtain a path $P_{i}$ which connects $x_{i}$ and $y_{i}$. Moreover, These $t$ paths are pairwise disjoint, so $\left\{P_{i}\right\}$ is a path collection. Thus, we may assume that $\kappa(G) \geq 2 t \epsilon n$.

Since $\delta(G) \geq \kappa(G) \geq 2 t \epsilon n$, we may create $t$ vertex disjoint paths $Q_{1}$, $Q_{2}, \ldots, Q_{t}$ in $G-\left\{y_{1}, y_{2}, \ldots, y_{t}\right\}$ such that $\left|Q_{i}\right|=2 \epsilon n-9$ which starts at $x_{i}$ for any $1 \leq i \leq t$. For $1 \leq i \leq t$, let $z_{i}$ be an end vertex of $Q_{i}$ other than $x_{i}$, and let $\mathscr{Q}=\bigcup_{i=1}^{t} V\left(Q_{i}\right)$. Notice $|\mathscr{Q}| \leq \kappa(G)-9 t$ so $G-\left(\mathscr{Q}-\left\{z_{1}, z_{2}, \ldots, z_{t}\right\}\right)$ is at least $10 t$-connected. By Theorem 9 , we know $G-\left(\mathscr{Q}-\left\{z_{1}, z_{2}, \ldots, z_{t}\right\}\right)$ is $t$-linked. This implies that we may link $z_{i}$ and $y_{i}$, and obtain a path collection $P_{1}, P_{2}, \ldots, P_{t}$, where $P_{i}$ is a path combining $Q_{i}$ and the link between $z_{i}$ and $y_{i}$.

By Lemma 1, this completes the proof of Lemma 4.

\section{Proof of Theorem 4}

First, we apply Lemma 4 to obtain a spanning path collection $\mathscr{P}=\left\{P_{1}, \ldots\right.$, $\left.P_{t}\right\}$. Take such a spanning path collection $\mathscr{P}$ with $\mu(\mathscr{P})$ minimum and suppose $\mathscr{P}$ is not desired.

If for any subcollection $\mathscr{A}$ of $\mathscr{P}, e(\mathscr{A}, \mathscr{P}-\mathscr{A}) \geq \frac{\epsilon^{2}}{24 \cdot 48} n^{2}$, then we may apply Lemma 2 to find a collection of paths $\mathscr{P}^{\prime}$ with $\mu\left(\mathscr{P}^{\prime}\right)<\mu(\mathscr{P})$. This contradicts our assumptions on $\mathscr{P}$.

So there exists a subcollection $\mathscr{A}$ of $\mathscr{P}$ with $e(\mathscr{A}, \mathscr{P}-\mathscr{A})<\frac{\epsilon^{2}}{24 \cdot 48} n^{2}$. Letting $c_{1}:=\frac{\epsilon}{24}$ and $c_{2}:=\frac{\epsilon}{75}$. Since $\sum_{P_{i} \in \mathscr{A}}\left|P_{i}\right| \geq \epsilon n \geq\left(4 c_{1}+c_{2}\right) n$ and $\sum_{P_{i} \in \mathscr{P}-\mathscr{A}}\left|P_{i}\right| \geq \epsilon n \geq\left(4 c_{1}+c_{2}\right) n$, we can apply Lemma 3 to find a spanning desired path collection $\mathscr{P}^{\prime}$, thus completing the proof.

\section{Proof of Theorem 6}

First we consider a matching $\mathscr{M}$ with $t$ edges such that each edge contains a vertex in $S$. Since $\sigma_{2}(G) \geq n+2 t-2$, we know $\delta(G) \geq 2 t$, and hence there exists such a matching $\mathscr{M}$. Let $\mathscr{M}:=\left\{x_{1} y_{1}, x_{2} y_{2}, \ldots, x_{t} y_{t}\right\}$ and let $S^{\prime}:=S \cup\left\{y_{i}: 1 \leq i \leq t\right\}$. If $G$ is $(2 t+1)$-connected, we can apply Theorem 4 for $S^{\prime}$. Then $G$ contains a spanning collection $\mathscr{P}$ of vertex disjoint paths $P_{i}=x_{i}, \ldots, y_{i}$ such that $\left(\gamma_{i}-\epsilon\right) n<\left|P_{i}\right|<\left(\gamma_{i}+\epsilon\right) n$ for all $1 \leq i \leq t$. In this case, $\left\{C_{1}, C_{2}, \ldots, C_{t}\right\}$ is a desired spanning collection of vertex disjoint cycles, whehe $C_{i}:=P_{i} \cup\left\{x_{i} y_{i}\right\}$. So we may assume that $G$ has a cut set $T$ of order $2 t$. 
By the degree sum condition, we know that $G-T$ has exactly two components and both of them are cliques. In this case, we can easily find a desired spanning collection of vertex disjoint cycles.

\section{References}

[1] G. Chartrand and L. Lesniak (2005). Graphs \& Digraphs, Fourth ed. Chapman \& Hall/CRC, Boca Raton, FL. MR2107429 (2005g:05001)

[2] Y. Chen, F. Tian and B. Wei (2001). Degree sums and path-factors in graphs. Graphs Combin. 17 61-71. MR1828628 (2002c:05126)

[3] G. A. Dirac (1952). Some theorems on abstract graphs. Proc. London Math. Soc. (3) 2 69-81. MR0047308 (13,856e)

[4] Y. Egawa, R. J. Faudree, E. Györi, Y. Ishigami, R. H. Schelp and H. Wang (2000). Vertex-disjoint cycles containing specified edges. Graphs Combin. 16 81-92. MR1750458 (2001a:05084)

[5] M. H. El-Zahar (1984). On circuits in graphs. Discrete Math. 50 227230. MR753712 (85g:05092)

[6] H. Enomoto (2001). Graph partition problems into cycles and paths. Discrete Math. 233 93-101. Graph theory (Prague, 1998). MR1825604 (2001m:05206)

[7] H. Enomoto and K. Ota (2000). Partitions of a graph into paths with prescribed endvertices and lengths. J. Graph Theory 34 163-169. MR1760421 (2000m:05181)

[8] R. Faudree, R. Gould, M. Jacobson and C. Magnant (2012). Distributing Vertices on a Hamiltonian Cycle. J. Graph Theory 69 28-45. MR2864621

[9] R. Johansson (1998). An El-Zahár type condition ensuring path-factors. J. Graph Theory 28 39-42. MR1619820 (99c:05114)

[10] K. Kawarabayashi (2002). Graph partition into paths containing specified vertices. Discrete Math. 248 271-277. MR1892703 (2003a:05120)

[11] C. Magnant and D. M. Martin (2010). An asymptotic version of a conjecture by Enomoto and Ota. J. Graph Theory 64 37-51. MR2656748 (2011h:05204)

[12] K. Menger (1927). Über reguläre Baumkurven. Math. Ann. 96 572-582. MR1512340 
Partitioning graphs into paths or cycles of prescribed lengths 161

[13] O. Ore (1960). Note on Hamilton circuits. Amer. Math. Monthly 6755. MR0118683 (22 \#\#9454)

[14] O. Ore (1963). Hamilton connected graphs. J. Math. Pures Appl. (9) 42 21-27. MR0146816 (26 \#\#4336)

[15] L. Pósa (1963). On the circuits of finite graphs. Magyar Tud. Akad. Mat. Kutató Int. Közl. 8 355-361 (1964). MR0197355 (33 \#\#5520)

[16] R. Thomas and P. Wollan (2005). An improved linear edge bound for graph linkages. European J. Combin. 26 309-324. MR2116174 (2005h:05122)

[17] J. E. Williamson (1977). Panconnected graphs. II. Period. Math. Hungar. 8 105-116. MR0463037 (57 \#\#3001)

Colton Magnant

Georgia Southern University

StATESBoro

GA 30460-8093

USA

E-mail address: dr.colton.magnant@gmail.com

Kenta OzeKI

National Institute for Informatics

TOKYO 101-8430

JAPAN

E-mail address: ozeki@comb.math.keio.ac.jp

Received January 15, 2011 